PAPER • OPEN ACCESS

\section{Numerical modelling of dynamic resistance in high-temperature superconducting coated- conductor wires}

To cite this article: Mark D Ainslie et al 2018 Supercond. Sci. Technol. 31074003

View the article online for updates and enhancements.
Related content

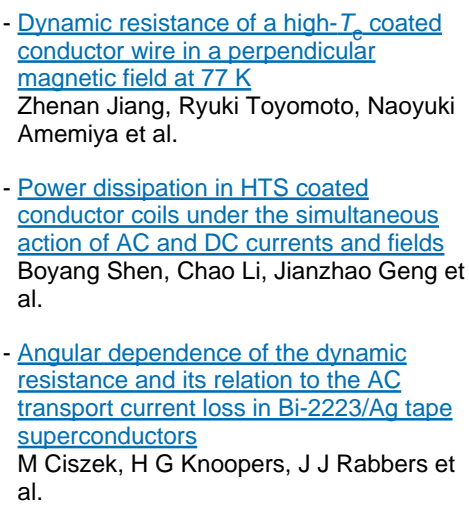

- Power dissipation in HTS coated conductor coils under the simultaneous action of AC and DC currents and fields Boyang Shen, Chao Li, Jianzhao Geng et al.

Anqular dependence of the dynamic resistance and its relation to the $A C$ transport current loss in $\mathrm{Bi}-2223 / \mathrm{Ag}$ tape superconductors

M Ciszek, H G Knoopers, J J Rabbers et al.

\section{Recent citations}

- Behaviour prediction of closed-loop HTS
$\frac{\text { coils in non-uniform AC fields }}{\text { Zhuoyan Zhong et al }}$
- Calculating the full-range dynamic loss of
$\frac{\text { HTS wires in an instant }}{\text { Francesco Grilli }}$
- Finite-element modelling of no-insulation
$\frac{\text { HTS coils using rotated anisotropic }}{\text { resistivity }}$
R C Mataira et al




\title{
Numerical modelling of dynamic resistance in high-temperature superconducting coated-conductor wires
}

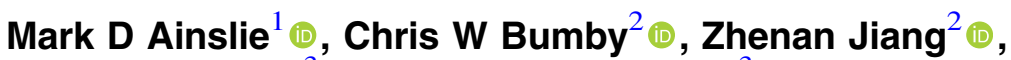 \\ Ryuki Toyomoto ${ }^{3}$ and Naoyuki Amemiya ${ }^{3}$ (i) \\ ${ }^{1}$ Bulk Superconductivity Group, Department of Engineering, University of Cambridge, United Kingdom \\ ${ }^{2}$ Robinson Research Institute, Victoria University of Wellington, New Zealand \\ ${ }^{3}$ Department of Electrical Engineering, Graduate School of Engineering, Kyoto University, Japan \\ E-mail: mark.ainslie@eng.cam.ac.uk
}

Received 13 March 2018, revised 25 April 2018

Accepted for publication 2 May 2018

Published 6 June 2018

\begin{abstract}
The use of superconducting wire within AC power systems is complicated by the dissipative interactions that occur when a superconductor is exposed to an alternating current and/or magnetic field, giving rise to a superconducting AC loss caused by the motion of vortices within the superconducting material. When a superconductor is exposed to an alternating field whilst carrying a constant DC transport current, a DC electrical resistance can be observed, commonly referred to as 'dynamic resistance.' Dynamic resistance is relevant to many potential hightemperature superconducting (HTS) applications and has been identified as critical to understanding the operating mechanism of HTS flux pump devices. In this paper, a 2D numerical model based on the finite-element method and implementing the $\boldsymbol{H}$-formulation is used to calculate the dynamic resistance and total AC loss in a coated-conductor HTS wire carrying an arbitrary DC transport current and exposed to background AC magnetic fields up to $100 \mathrm{mT}$. The measured angular dependence of the superconducting properties of the wire are used as input data, and the model is validated using experimental data for magnetic fields perpendicular to the plane of the wire, as well as at angles of $30^{\circ}$ and $60^{\circ}$ to this axis. The model is used to obtain insights into the characteristics of such dynamic resistance, including its relationship with the applied current and field, the wire's superconducting properties, the threshold field above which dynamic resistance is generated and the flux-flow resistance that arises when the total driven transport current exceeds the field-dependent critical current, $I_{\mathrm{c}}(\boldsymbol{B})$, of the wire. It is shown that the dynamic resistance can be mostly determined by the perpendicular field component with subtle differences determined by the angular dependence of the superconducting properties of the wire. The dynamic resistance in parallel fields is essentially negligible until $J_{c}$ is exceeded and flux-flow resistance occurs.
\end{abstract}

Keywords: dynamic resistance, AC loss, coated conductor, finite-element method, numerical simulation, critical current density (superconductivity)

(Some figures may appear in colour only in the online journal)

\section{Introduction}

Original content from this work may be used under the terms of the Creative Commons Attribution 3.0 licence. Any further distribution of this work must maintain attribution to the author(s) and the title of the work, journal citation and DOI.
Recent advances in the manufacturing of high-temperature superconducting (HTS) wire mean that long lengths $(\mathrm{km}+)$ of 'coated-conductor' ReBCO wire are now commercially available. This has sparked new interest in a range of new 
large-scale applications for superconducting magnets and power systems, which exploit the higher operating temperatures and associated thermal 'robustness' inherent to HTS materials. In particular, the development of AC power system applications, such as transformers, motors and generators, is an area of expanding interest.

However, the use of superconducting wire within an $\mathrm{AC}$ power system is complicated by the dissipative interactions that occur when a superconductor is exposed to an alternating current and/or magnetic field. This gives rise to a superconducting AC loss, caused by the motion of vortices within the superconducting material: 'magnetisation loss' describes dissipation due to the purely magnetic hysteresis behaviour of a superconducting body carrying zero transport current and 'transport loss' describes dissipation which occurs when an alternating transport current imposes an alternating self-field on a conductor, in the absence of an applied field. In practical applications, a cryogenic cooling system must extract the resulting heat load in order to enable constant temperature operation, and this means a comprehensive understanding of the mechanism and magnitude of $\mathrm{AC}$ losses is extremely important to the design and development of new superconducting magnets and rotating machines.

AC loss also arises when a superconductor is exposed to an alternating field whilst carrying a constant DC transport current. In this case, a DC electrical resistance is observed, commonly referred to as 'dynamic resistance' [1-4]. This situation is relevant to many potential HTS applications, including superconducting synchronous machines, NMR magnets and other unshielded DC magnet applications, and this dynamic resistance been identified as critical to understanding the operating mechanism of HTS flux pump devices [5-9]. Dynamic resistance, $R_{\mathrm{dyn}}$, can be measured using simple electrical techniques, and gives rise to a dissipative loss $Q_{\mathrm{dyn}}=I_{\mathrm{dc}}^{2} R_{\mathrm{dyn}}$, where $I_{\mathrm{dc}}$ is the DC transport current. However, in this situation, there also exist simultaneous magnetisation losses within the wire that are not probed by this particular electrical measurement. The total loss is a combination of the dynamic resistance and magnetisation terms, and is difficult to measure experimentally, due to additional dissipation associated with the DC current injection.

In this paper, a $2 \mathrm{D}$ numerical model based on the finiteelement method and implementing the $\boldsymbol{H}$-formulation is used to calculate the dynamic resistance and total AC loss in a coated-conductor HTS wire carrying an arbitrary DC transport current and exposed to background AC magnetic fields up to $100 \mathrm{mT}$. The measured angular dependence of the superconducting properties of wire, $I_{\mathrm{c}}(B, \theta)$, and $n$-value, $n(B, \theta)$, for the $E-J$ power law representing the superconductor's electrical resistivity, are used as input data, and the model is validated using measured experimental data for external AC magnetic fields applied perpendicular to the plane of the wire, as well as at angles of $30^{\circ}$ and $60^{\circ}$. The model is used to obtain insights into the particular characteristics of such dynamic resistance, including its relationship with the applied current and field, the superconducting properties of the wire, the threshold field above which dynamic resistance is generated and the flux-flow resistance

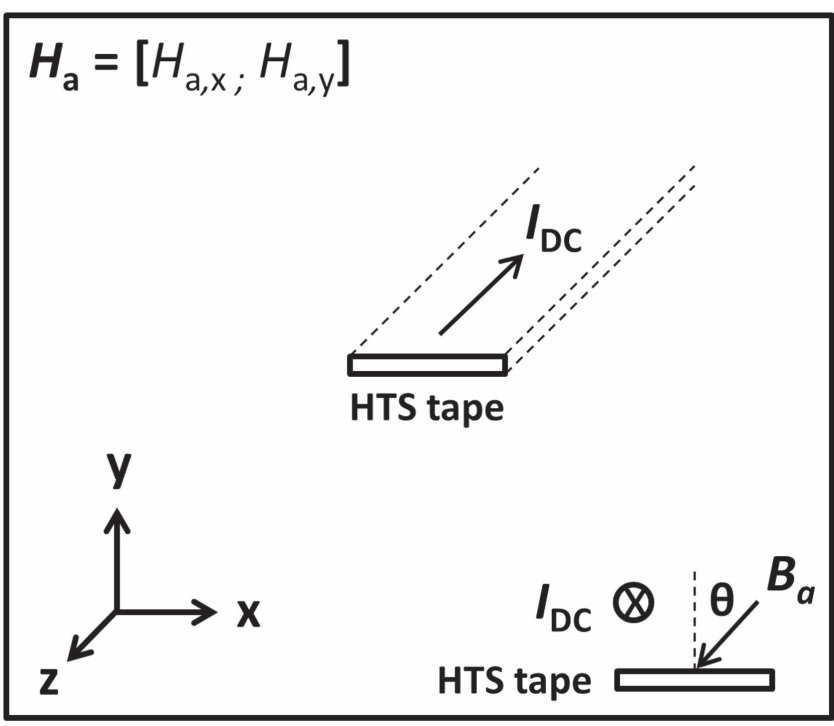

Figure 1. 2D infinite long numerical model geometry used to calculate the dynamic resistance of the HTS coated-conductor wire. The instantaneous external AC magnetic field applied to the wire at frequency $f$ is $\boldsymbol{B}_{a}(t)=\boldsymbol{B}_{\mathrm{app}} \cdot \sin (2 \pi f t)$. $I_{\mathrm{DC}}$ is the DC transport current flowing along the wire. $H_{a, x}, H_{a, y}$ are the Cartesian components of $\boldsymbol{H}_{a}=\boldsymbol{B}_{a} / \mu_{0}$.

that arises when the total driven transport current exceeds the field-dependent critical current, $I_{\mathrm{c}}(\boldsymbol{B})$, of the wire.

\subsection{Numerical modelling framework}

The 2D infinitely long numerical model, shown in figure 1 , is based on the $\boldsymbol{H}$-formulation [10-16], where the governing equations are derived from Maxwell's equations: Faraday's law (1) and Ampere's law (2).

$$
\begin{gathered}
\nabla \times \boldsymbol{E}+\frac{\mathrm{d} \boldsymbol{B}}{\mathrm{d} t}=\nabla \times \boldsymbol{E}+\frac{\mathrm{d}\left(\mu_{0} \mu_{r} \boldsymbol{H}\right)}{\mathrm{d} t}=0, \\
\nabla \times \boldsymbol{H}=\boldsymbol{J},
\end{gathered}
$$

where $\boldsymbol{H}=\left[H_{x}, H_{y}\right]$ represents the components of the magnetic field strength, $\boldsymbol{J}=\left[J_{z}\right]$ represents the current density and $\boldsymbol{E}=\left[E_{z}\right]$ represents the electric field. $\mu_{0}$ is the permeability of free space, and for the superconducting layer and surrounding sub-domain (simply assumed to be air), the relative permeability is simply $\mu_{r}=1$. Only the superconducting layer of the wire is modelled, assuming that eddy current losses within the wire architecture (e.g., substrate, stabiliser layers) is negligible: a reasonable assumption for the wire at power frequencies $(\sim 50 / 60 \mathrm{~Hz})[17,18]$. Since the substrate of the wire under investigation is non-magnetic, consideration of any ferromagnetic losses, and indeed the influence of a magnetic substrate on the superconducting properties, is also unnecessary. Isothermal conditions are assumed and a constant temperature, $T=77 \mathrm{~K}$; hence, no thermal model is included. 


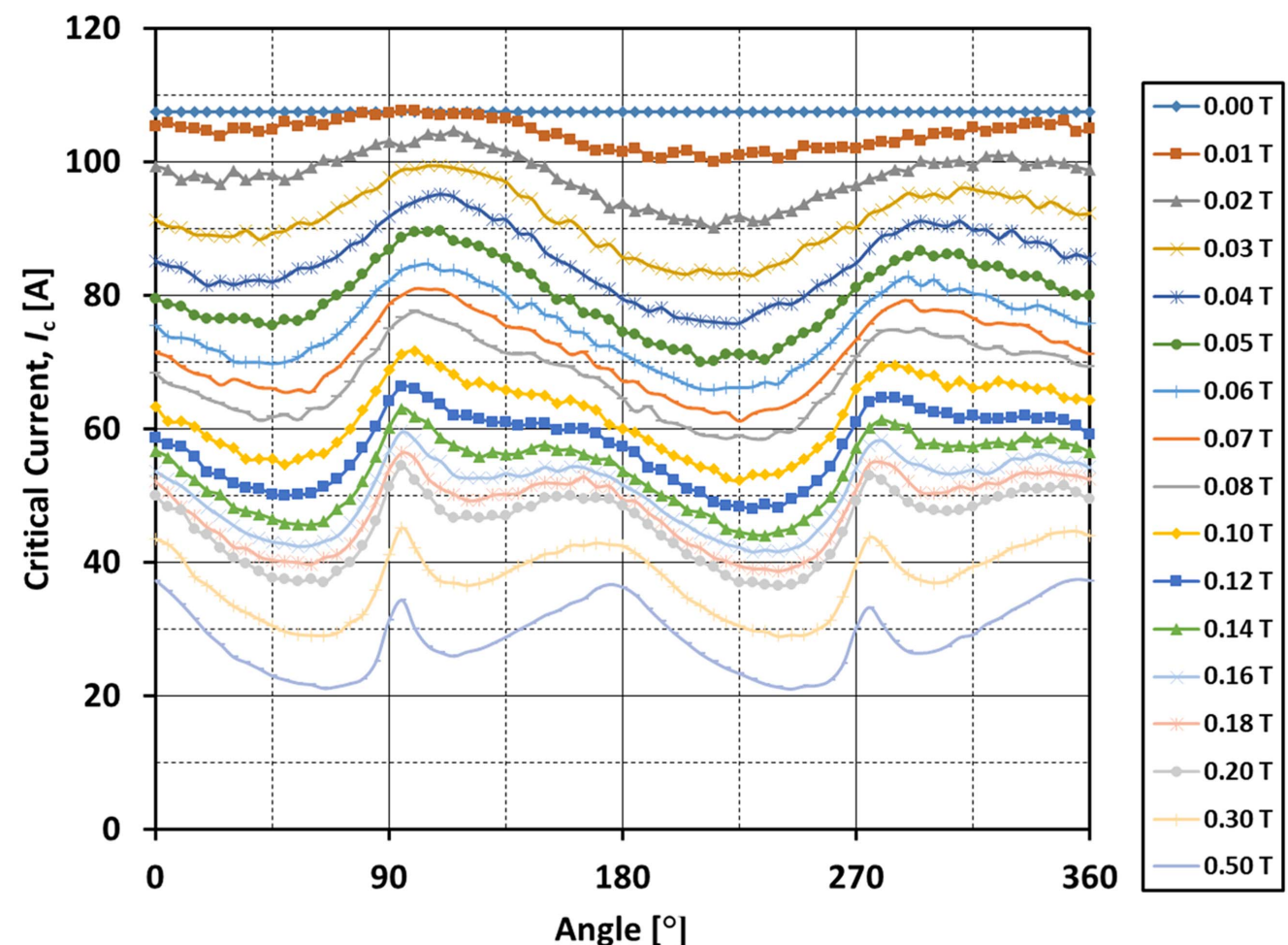

Figure 2. Experimental $I_{\mathrm{c}}(B, \theta)$ data used in the $\boldsymbol{H}$-formulation model. Data was measured at $77 \mathrm{~K}$ in magnetic fields up to $0.5 \mathrm{~T}$ from a short sample of SuperPower's SCS4050-AP wire. The $J_{\mathrm{c}}(B, \theta)$ data is corrected for self-field using the technique presented in [21] and input into the model using a two-variable, direct interpolation $[25,26]$.

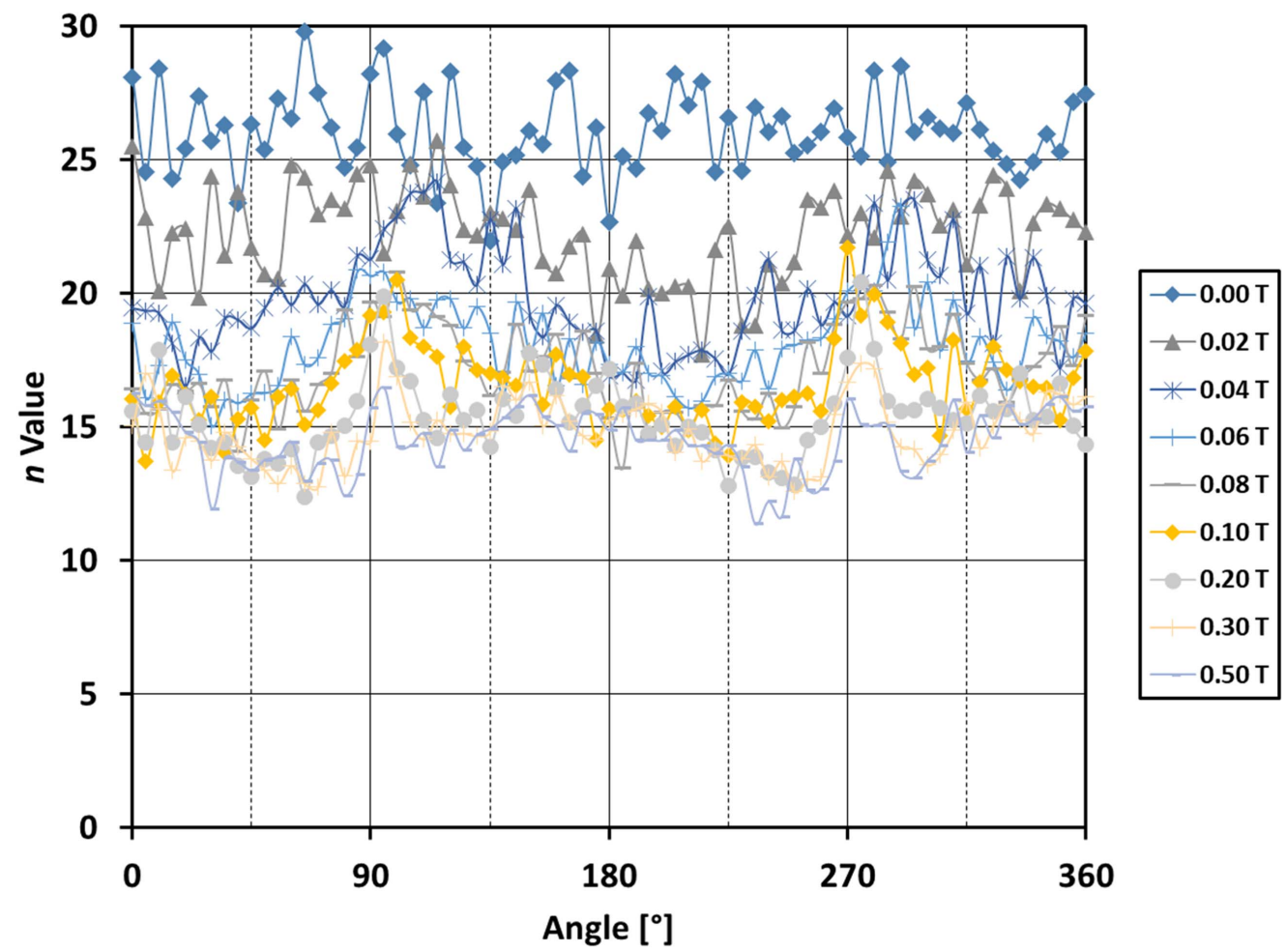

Figure 3. Experimental $n(B, \theta)$ data used in the model, measured at $77 \mathrm{~K}$ in magnetic fields up to $0.5 \mathrm{~T}$ from a short sample of SuperPower's SCS4050-AP wire. The data is input into the model using a two-variable, direct interpolation $[25,26]$. 


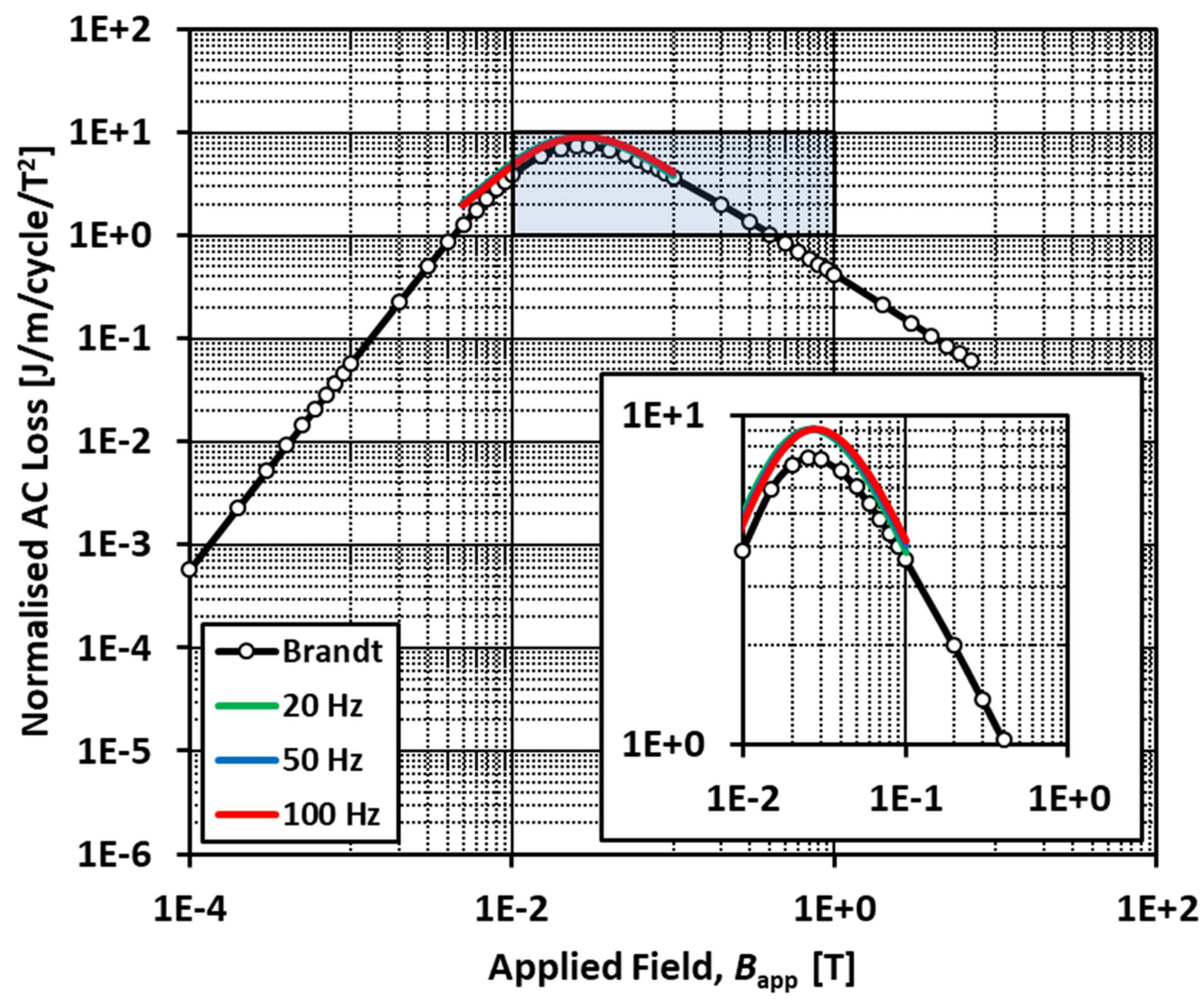

Figure 4. Comparison of calculated magnetisation AC loss using the numerical model for perpendicular magnetic fields up to $100 \mathrm{mT}$ and analytical results according to the Brandt equation [36]. The analytical calculation uses $J_{\mathrm{c} 0}=2.6875 \times 10^{10} \mathrm{~A} \mathrm{~m}^{-2}$, which corresponds to the measured $I_{\mathrm{c}}(77 \mathrm{~K}$, self-field $)=107.5 \mathrm{~A}$ and a width of $4 \mathrm{~mm}$ and thickness $1 \mu \mathrm{m}$ for the superconducting layer. The results are normalised with respect to $\left|\boldsymbol{B}_{\text {app }}\right|^{2}$.

It is assumed that electric field $E_{z}$ is parallel to the current density $J_{z}$ and the electrical properties of the superconductor are modelled by the $E-J$ power law $[19,20]$ in (3).

$$
\boldsymbol{E}=\frac{E_{0}}{J_{\mathrm{c}}(B, \theta)}\left|\frac{J}{J_{\mathrm{c}}(B, \theta)}\right|^{n-1} \boldsymbol{J},
$$

where $E_{0}$ is the characteristic electric field, $1 \mu \mathrm{V} \mathrm{cm}{ }^{-1}, B$ is magnitude of the local magnetic flux density and $\theta$ is the angular direction of that field as defined in figure 1.

Traditionally, the relationship between the critical current density $J_{\mathrm{c}}(B, \theta)$ and the critical current $I_{\mathrm{c}}(B, \theta)$ is given by (4), where $S$ is the cross-sectional area of the superconducting layer. However, this direct approach does not include selffield effects, i.e., $B$ refers to the external applied field, rather than the local magnetic flux density in (3). This can introduce numerical errors when the applied field is comparable to the self-field. Since, in this paper, the applied fields are $100 \mathrm{mT}$ or less, the self-field is taken into account when calculating $J_{\mathrm{c}}(B, \theta)$ based on the technique presented in [21], and using the example model files available at [22], which obtains $J_{\mathrm{c}}(B, \theta)$ based on the local magnetic flux density, rather than the external applied field using (4). The width of the tape is $4 \mathrm{~mm}$ and the thickness of the superconducting layer is $1 \mu \mathrm{m}$; however, the homogenisation technique presented in [23, 24] is used to improve the computational speed of the model without compromising accuracy, and the thickness of the superconducting layer in the model is artificially expanded to $100 \mu \mathrm{m}$.

$$
J_{\mathrm{c}}(B, \theta)=\frac{I_{\mathrm{c}}(B, \theta)}{S} .
$$

Experimentally measured values of $I_{\mathrm{c}}(B, \theta)$ (from which $J_{\mathrm{c}}(B, \theta)$ is calculated as described above) and $n(B, \theta)$ were used in the $\boldsymbol{H}$-formulation model. This data was obtained at $77 \mathrm{~K}$ in magnetic fields up to $0.5 \mathrm{~T}$, from a short sample of the same SuperPower SCS4050-AP wire as used in our dynamic resistance experiments. The experimental values, shown in figures 2 and 3, respectively, are input into the model using a two-variable, direct interpolation $[25,26]$, which is significantly faster than data fitting methods, yet without compromising accuracy. The measured, self-field critical current, $I_{\mathrm{c} 0}$, of the wire at $77 \mathrm{~K}$ is $107.5 \mathrm{~A}$. In figure 3 , there is some scatter in the calculated $n$-values after fitting equation (3) to the experimental data, most noticeable for the $0 \mathrm{~T}$ data where a scatter of $n \pm 2-3$ is observed. The $n$-value fit is sensitive to the curvature of the experimental data above $J_{\mathrm{c}}$, a regime where the experimental data is also vulnerable to the influence of resistive heating. However, at higher fields, this scatter is substantially depressed and the systematic 'double-peak' in the $n$-value is clear, following the measured $I_{\mathrm{c}}$ data in figure 2 . 


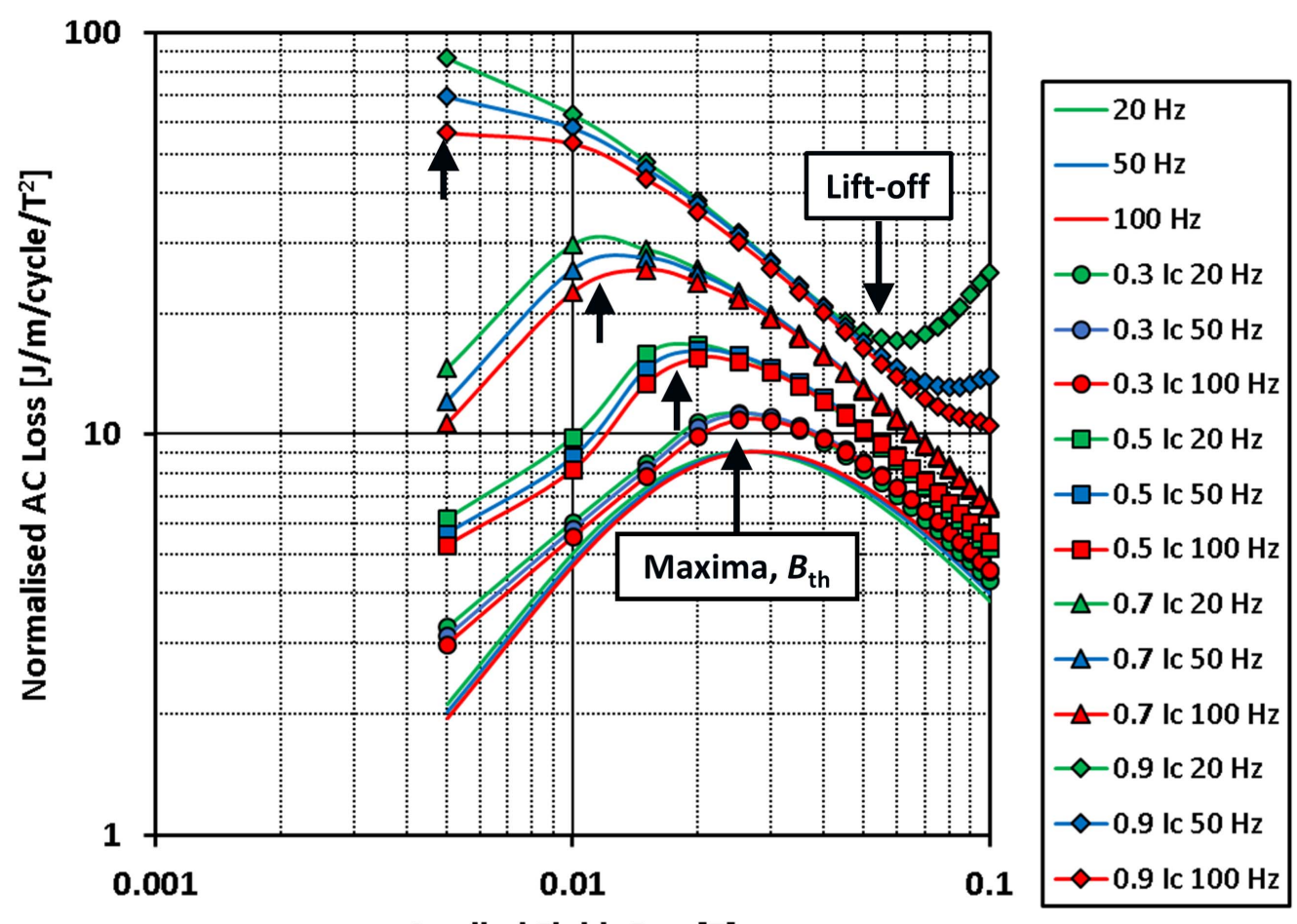

Applied Field, $B_{\text {app }}[T]$

Figure 5. Calculated total AC loss, which includes the contribution of the magnetisation loss, $Q_{\text {mag }}$, and the contribution from the dynamic resistance, $Q_{\mathrm{dyn}}$, as described by (6), using the numerical model for perpendicular magnetic fields up to $100 \mathrm{mT}$ and DC transport currents corresponding to $I_{\mathrm{DC}} / I_{\mathrm{c}}=0.3,0.5,0.7$ and 0.9 . The case from figure 4 when no DC transport current is applied, i.e., $I_{\mathrm{DC}} / I_{\mathrm{c}}=0$, is also shown for comparison. The results are normalised with respect to $\left|\boldsymbol{B}_{\text {app }}\right|^{2}$.

A mapped mesh (200x-elements along the width, $10 y$ elements along the thickness, and a linear element distribution with an element ratio of 3 between the central elements and the edge and top/bottom surface elements) is used in the superconducting layer to decrease the number of mesh elements [27], while still retaining enough mesh elements at the top and bottom of the wire to accurately simulate the current density associated with the DC current, and a free triangular mesh is used in the surrounding sub-domain. Linear, curlconforming elements are employed for the entire model. For the non-superconducting sub-domain surrounding the superconducting layer, a linear Ohm's law is considered, $\boldsymbol{E}=\rho \boldsymbol{J}$, where $\rho=1 \Omega \mathrm{m}$ is a specific, high constant resistivity.

An integral constraint is applied to the superconducting layer to represent the DC transport current flowing in the superconducting tape. A transport current, $I$, through the cross-section $\boldsymbol{S}$ of the tape is therefore described by (5).

$$
I=\int \boldsymbol{J} \cdot \mathrm{d} \boldsymbol{S}=I_{\mathrm{app}}(t),
$$

where $I_{\text {app }}(t)$ is a ramp function of ramp rate $10 \mathrm{~A} \mathrm{~s}^{-1}$ that is then held at its final value, $I_{\mathrm{DC}}$, which is defined by the ratio of $I_{\mathrm{DC}} / I_{\mathrm{c}}$. In this paper, the cases for $I_{\mathrm{DC}} / I_{\mathrm{c}}=0.3,0.5,0.7$ and 0.9 are examined, where $I_{\mathrm{c}}=107.5 \mathrm{~A}$ as defined earlier.

Once the appropriate $I_{\mathrm{DC}} / I_{\mathrm{c}}$ value is reached, $\mathrm{AC}$ magnetic fields up to $100 \mathrm{mT}$ in amplitude are applied in $5 \mathrm{mT}$ increments by setting appropriate boundary conditions for $H_{x}, H_{y}$ [28]: the instantaneous external AC magnetic field applied to the wire at frequency $f$ is $\boldsymbol{B}_{a}(t)=\boldsymbol{B}_{\text {app }} \cdot \sin (2 \pi f t)$, and $H_{a, x}, H_{a, y}$ are the Cartesian components of $\boldsymbol{H}_{a}=\boldsymbol{B}_{a} / \mu_{0}$, as shown in figure 1 . As such both $\theta=0^{\circ}$ and $\theta=180^{\circ}$ are perpendicular to the plane of the wire, but point in opposite directions, i.e., into top surface and substrate side of the wire, respectively.

The calculation of the total AC loss $\left(\mathrm{J} \mathrm{m}^{-1} /\right.$ cycle) includes separate contributions from the magnetisation loss, $Q_{\mathrm{mag}}$, and the dynamic resistance, $Q_{\mathrm{dyn}}$, and in the 2D infinitely long model of the superconducting tape, this can be expressed as (6).

Total AC loss $(\mathrm{J} /$ cycle $/ \mathrm{m})=\int_{0}^{T} \int \boldsymbol{E} \cdot \boldsymbol{J} \mathrm{d} S \mathrm{~d} t=Q_{\text {mag }}+Q_{\text {dyn }}$,

where $T$ is the period of one cycle. When computing this value, we have taken the integral result between $0.5 T$ and $1.5 T$, as this period does not include the transient, first half-cycle, where the superconductor is magnetised from its 'virgin' state [29].

In order to calculate the dynamic resistance, the average electric field across the entire conductor cross-section is firstly obtained using (7). The instantaneous dynamic resistance and associated power loss can then be obtained from (8) and (9), respectively, and the dynamic resistance loss, $Q_{\mathrm{dyn}}$, can be calculated from (10). Finally, the dynamic resistance, $R_{\mathrm{dyn}}$, in units of $\left(\Omega \mathrm{m}^{-1} /\right.$ cycle) can be found using (11).

$$
E_{\mathrm{ave}}(t)=\frac{\int_{S} \boldsymbol{E}(t) \cdot \mathrm{d} \boldsymbol{S}}{S},
$$




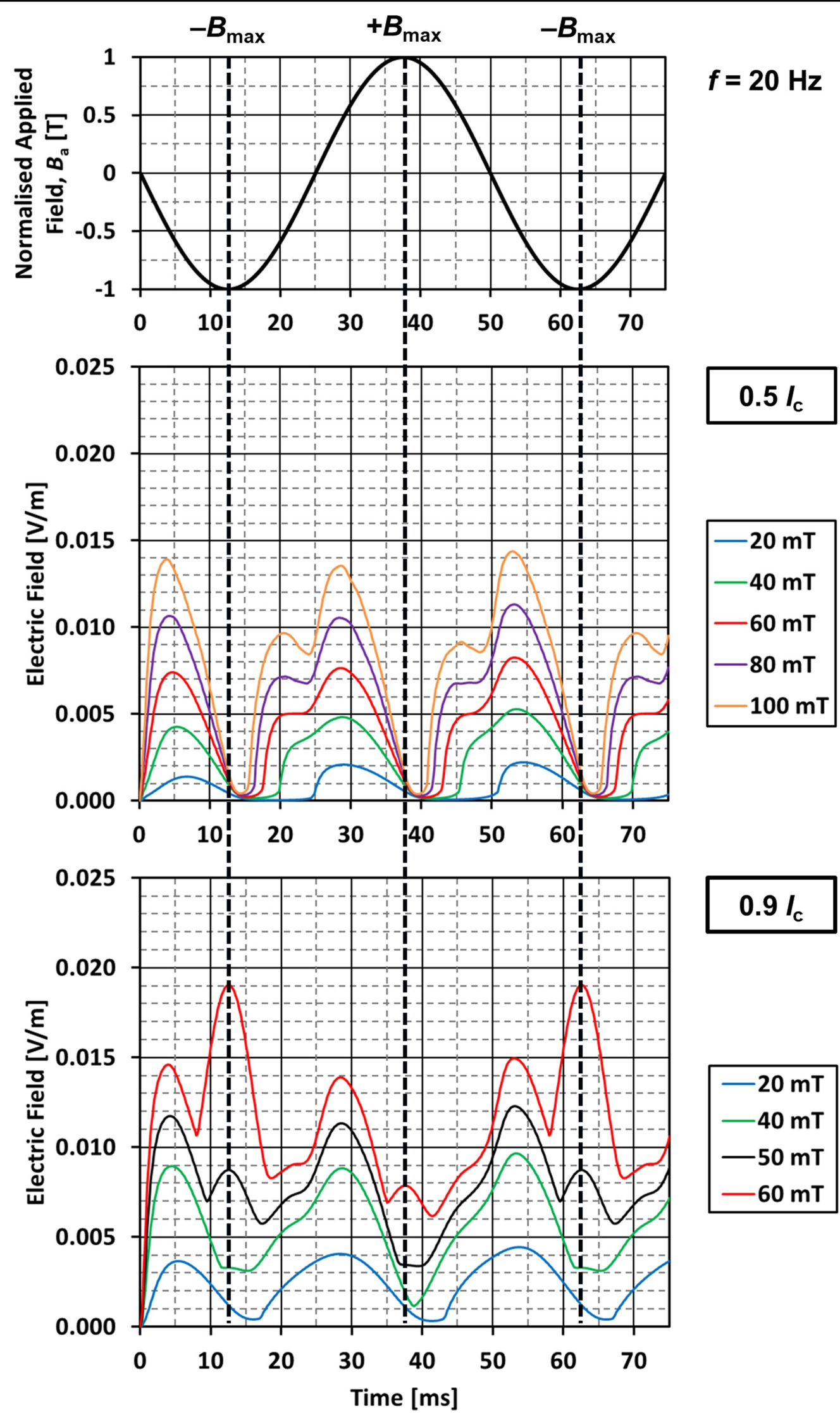

Figure 6. Calculated average electric field across the cross-section of the wire for $0.5 I_{\mathrm{c}}$ for perpendicular external AC magnetic fields with amplitude $\left|\boldsymbol{B}_{\text {app }}\right|=20,40,60,80$ and $100 \mathrm{mT}$ (middle figure); and 0.9I $I_{\mathrm{c}}$ for $\left|\boldsymbol{B}_{\text {app }}\right|=20,40,50$ and $60 \mathrm{mT}$ (bottom figure) for a frequency of $20 \mathrm{~Hz}$. The top figure shows the normalised $\boldsymbol{B}_{a}(t)$ with time, where $+B_{\max }$ corresponds to the peak of the applied field oriented towards the top surface $\left(\theta=0^{\circ}\right)$ and $-B_{\max }$ corresponds to the peak of the applied field oriented towards the bottom surface (substrate side; $\theta=180^{\circ}$ ). 
(a)
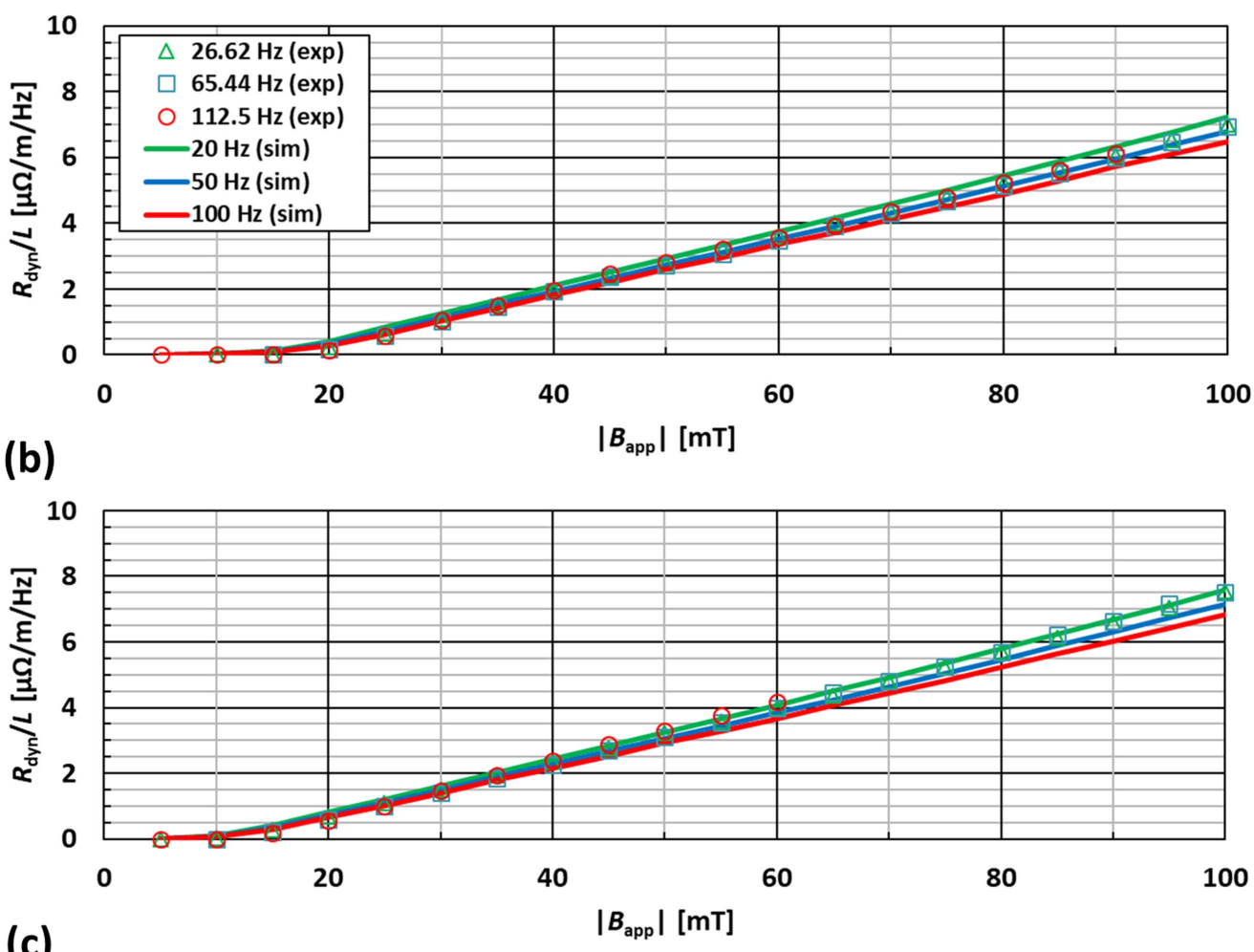

(c)

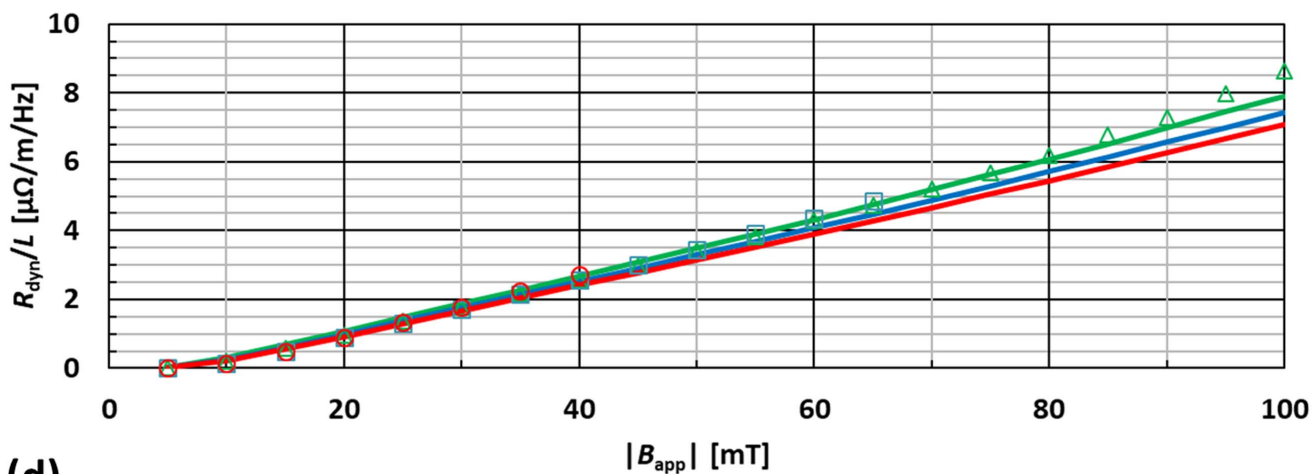

(d)

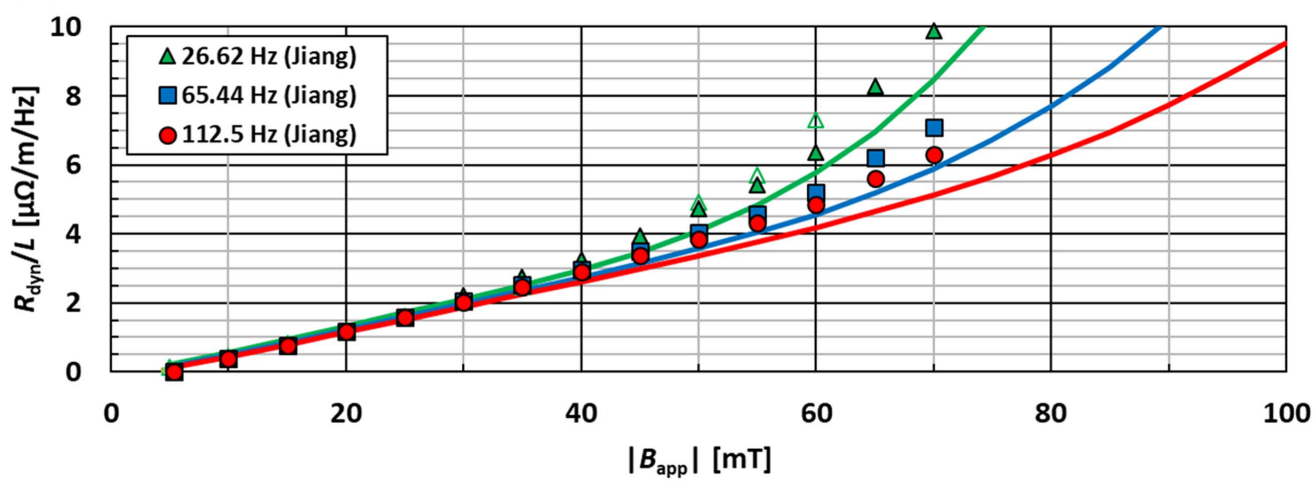

Figure 7. Comparison of the calculated dynamic resistance and experimental results for $I_{\mathrm{DC}} / I_{\mathrm{c}}=$ (a) 0.3 , (b) 0.5 , (c) 0.7 and (d) 0.9 for perpendicular external AC magnetic field amplitudes up to $100 \mathrm{mT}$ (in $5 \mathrm{mT}$ increments). The results are calculated for frequencies of 20,50 and $100 \mathrm{~Hz}$ and the experimental data corresponds to frequencies of 26.62, 65.44 and $112.5 \mathrm{~Hz}$.

$$
\begin{aligned}
& R_{\mathrm{dyn}}(t)=\frac{E_{\mathrm{ave}}(t)}{I(t)}=\frac{E_{\mathrm{ave}}(t)}{I_{\mathrm{dc}}}, \quad \text { (8) } \quad Q_{\mathrm{dyn}}\left(\mathrm{J} \mathrm{m}^{-1} / \text { cycle }\right)=\int_{0}^{T} I_{\mathrm{dc}}^{2} R_{\mathrm{dyn}}(t) \cdot \mathrm{d} t=I_{\mathrm{dc}}^{2} \int_{0}^{T} R_{\mathrm{dyn}}(t) \cdot \mathrm{d} t, \\
& P_{\mathrm{dyn}}(t)=I^{2}(t) R_{\mathrm{dyn}}(t)=I_{\mathrm{dc}}^{2} R_{\mathrm{dyn}}(t), \\
& R_{\mathrm{dyn}}\left(\Omega \mathrm{m}^{-1} / \text { cycle }\right)=Q_{\mathrm{dyn}} / I_{\mathrm{dc}}^{2}
\end{aligned}
$$




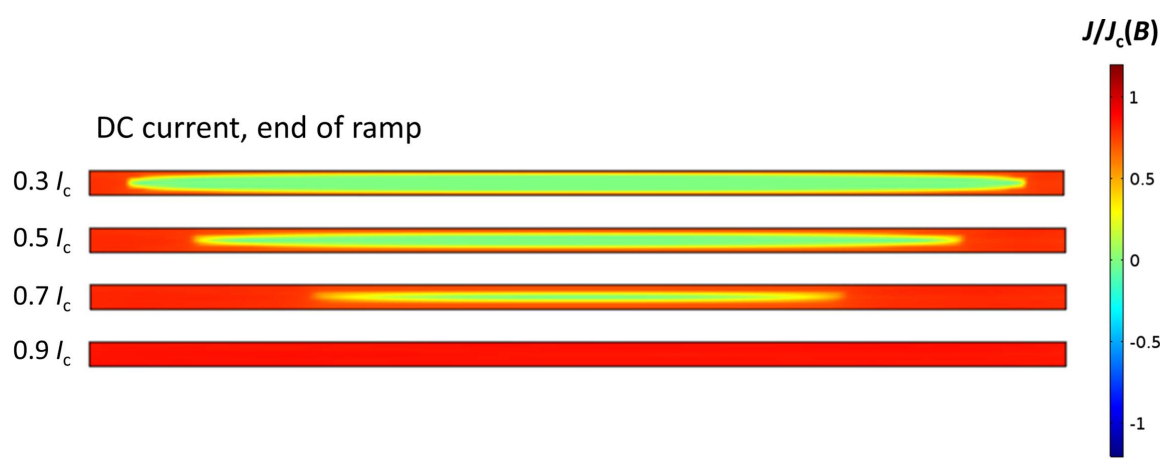

Figure 8. Calculated current density distributions, normalised by $J_{\mathrm{c}}(\boldsymbol{B})$, within the cross-section of the wire for the simple DC transport current cases (in the absence of an applied AC field) at the time when the ramped current reaches its final value as defined by $I_{\mathrm{DC}} / I_{\mathrm{c}}$.

(a) No dynamic resistance: $0.3 I_{c}, 20 \mathrm{~Hz}, B_{\text {app }}=10 \mathrm{mT}$

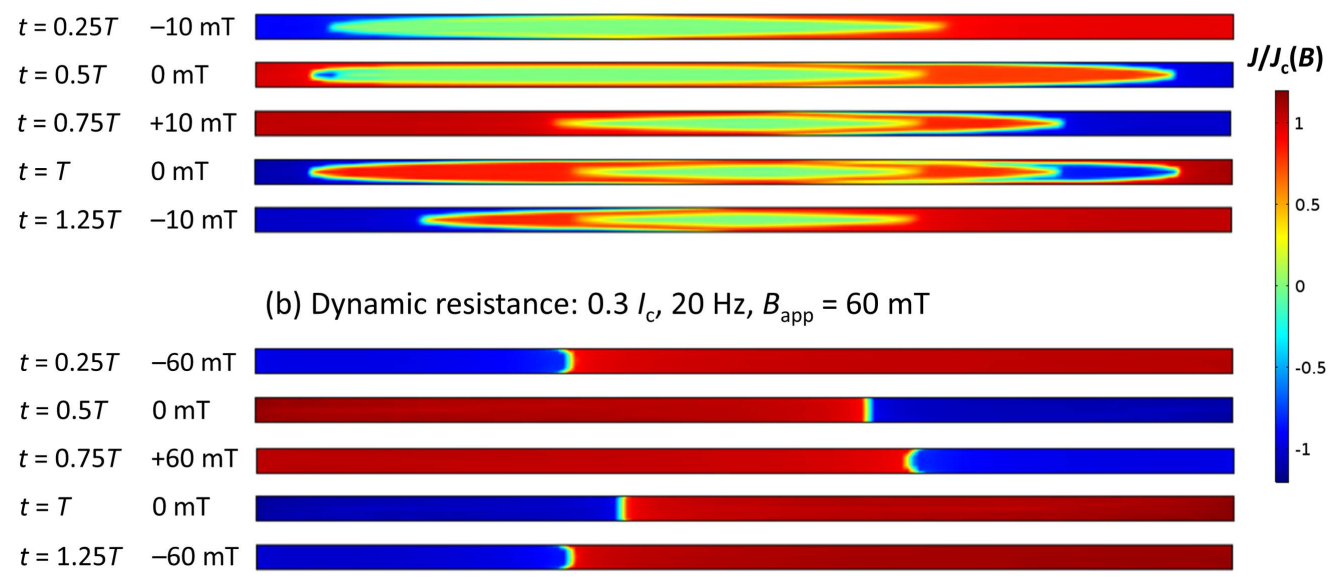

Figure 9. Calculated current density distributions, normalised by $J_{\mathrm{c}}(\boldsymbol{B})$, within the cross-section of the wire for perpendicular external AC magnetic fields when $I_{\mathrm{DC}}=0.3 I_{\mathrm{c}}$ and $f=20 \mathrm{~Hz}$. Data is shown for the instants at the positive and negative peaks of the applied field, as well as the zero-crossing. Two cases are shown: (a) $\left|\boldsymbol{B}_{\text {app }}\right|=10 \mathrm{mT}$ and (b) $\left|\boldsymbol{B}_{\text {app }}\right|=60 \mathrm{mT}$.

This approach removes ambiguity in defining the geometric boundaries between the transport and magnetisation currents throughout the AC cycle, and hence differs from previous analytical and finite-element approaches, which have considered dynamic resistance to arise only from current flowing in the central region of the wire [30-32].

\subsection{Experimental setup}

A detailed description of the experimental procedure for the dynamic resistance measurements can be found in [33]. An AC magnetic field was applied by a copper-wound electro-magnet that can generate up to $100 \mathrm{mT}$ peak magnetic field at frequencies up to $112.5 \mathrm{~Hz}$ [34]. The sample can be rotated inside the magnet, and the field angle, $\theta$, can thus be varied. A DC power supply was used to supply a DC transport current of up to $300 \mathrm{~A}$ to the SuperPower sample. Two voltage taps were soldered on the edges of the sample, and the leads were arranged in a spiral loop to minimise induced AC pick-up from the external magnetic field [35]. The distance between the voltage taps in these experiments was $5 \mathrm{~cm}$ and the time-averaged DC voltage across the voltage taps was measured using a Keithley 2182 nanovoltmeter.

\section{Model verification and experimental results}

\subsection{Perpendicular applied magnetic fields}

2.1.1. Total AC loss. Firstly, the magnetisation AC loss of the wire is calculated for the case where the DC transport current is zero and an external AC magnetic field is applied perpendicular to the plane of the wire, i.e., $\theta=0^{\circ}$. Figure 4 shows the calculated magnetisation AC loss, normalised by $\left|\boldsymbol{B}_{\text {app }}\right|^{2}$ (where $\boldsymbol{B}_{\text {app }}=\mu_{0} \boldsymbol{H}_{\text {app}}$ ), of the wire for magnetic fields up to $100 \mathrm{mT}$ and for frequencies of 20,50 and $100 \mathrm{~Hz}$, as calculated by the numerical model and compared with Brandt's analytical equation [36]. It is shown that the numerical model calculation is slightly higher than the analytical result due to our use of measured, real $J_{\mathrm{c}}(\boldsymbol{B})$ 


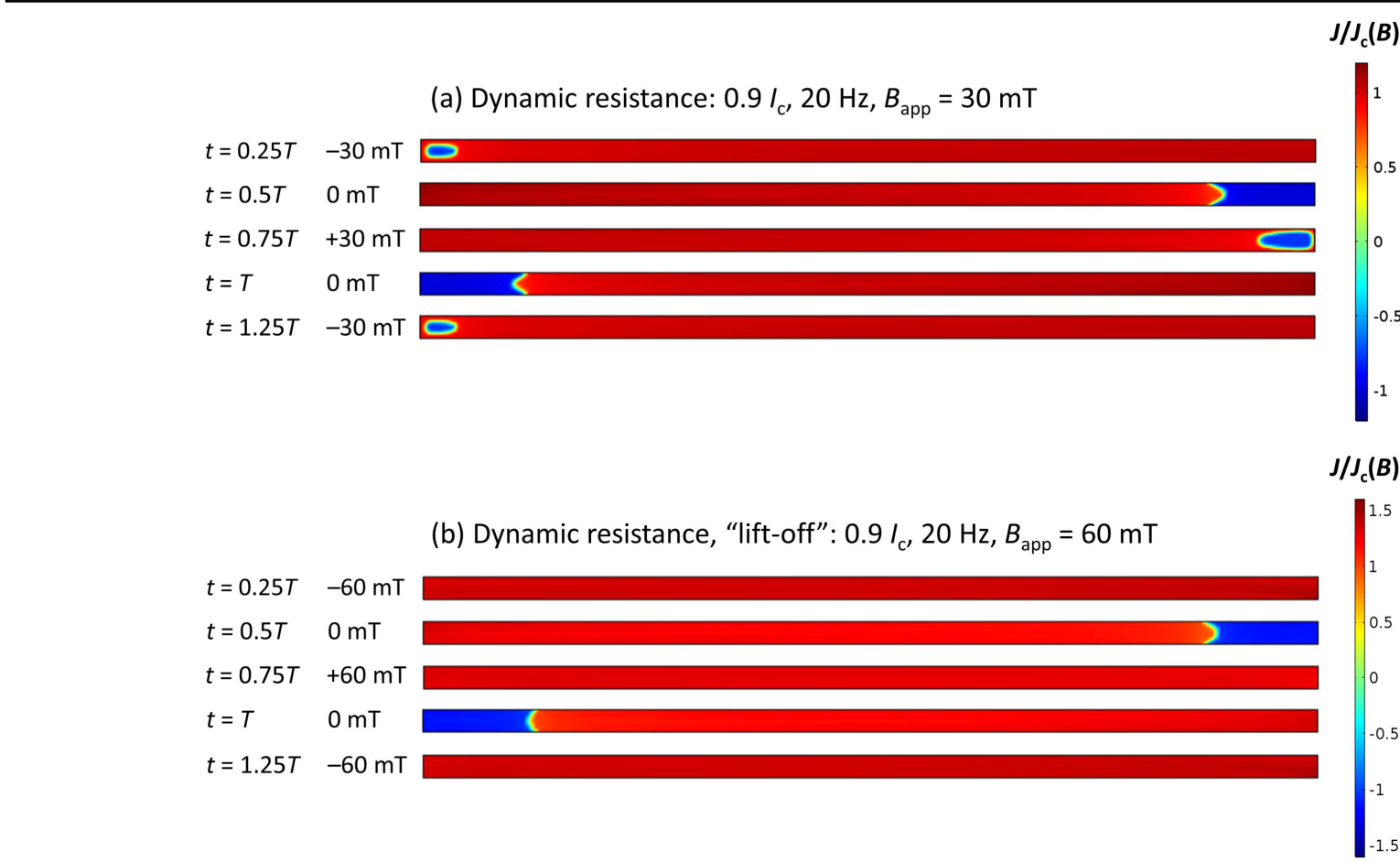

Figure 10. Calculated current density distributions, normalised by $J_{\mathrm{c}}(\boldsymbol{B})$, within the cross-section of the wire for perpendicular external AC magnetic fields when $I_{\mathrm{DC}}=0.9 I_{\mathrm{c}}$ and $f=20 \mathrm{~Hz}$. Data is shown for the instants at the positive and negative peaks of the applied field, as well as the zero-crossing. Two cases are shown: (a) $\left|\boldsymbol{B}_{\text {app }}\right|=30 \mathrm{mT}$ and (b) $60 \mathrm{mT}$. Case (a) corresponds to the linear dynamic resistance regime, and case (b) corresponds to the 'lift-off' regime, where there exists an additional flux-flow resistance term because the total driven transport current exceeds the field-dependent $I_{\mathrm{c}}$ throughout the wire. Note the different scale used in (b), where the current density $J$ significantly exceeds the $J_{\mathrm{c}}$ of the wire.

characteristics, rather than the constant $J_{\mathrm{c}}$ approximation used in the Brandt equation. There is also a slight frequency dependence of the simulation result, which is commonly observed when using time-dependent models based on the $E-J$ power law [37-40]. The impact of this on the dynamic resistance calculations is discussed later in the paper. Despite these minor discrepancies, values calculated using equation (6) are broadly consistent with the Brandt expression, thus validating our numerical model. This also gives a base case to compare subsequent total AC loss calculations in which a DC transport current gives rise to loss components from both magnetisation loss and dynamic resistance.

The maxima of the normalised magnetisation AC loss in figure 4 correspond to the effective penetration field of the wire, as it denotes the point at which the shielding current capacity is effectively saturated [30]. For values of $I_{\mathrm{DC}}>0$, the maxima shift due to the superposition of the transport current and shielding (magnetisation) currents, with the effective penetration field decreasing with increasing values of $I_{\mathrm{DC}}$. This is shown clearly in figure 5 . As in the zero current case, these maxima similarly correspond to the threshold field, $B_{\text {th }}$, above which where there is no longer a shielded region inside the wire and hence dynamic resistance occurs. For the case $I_{\mathrm{DC}} / I_{\mathrm{c}}=0.9$, there are also minima turning points, such that for fields above these points, the normalised total AC loss begins to increase again. This relates to the emergence of an additional flux-flow resistance term that arises once the total driven transport current exceeds the fielddependent $I_{\mathrm{c}}$ of the wire [30]. In the following section, the relationship of these maxima and minima present in the total AC loss curves and the particular characteristics of the dynamic resistance curves are explained in more detail.

2.1.2. Dynamic resistance. In this section, the dynamic resistance is calculated using the numerical model based on (7)-(11). Figure 6 shows the calculated average electric field, using (7), across the cross-section of the wire for $0.5 I_{\mathrm{c}}$ under perpendicular external AC magnetic fields with $\left|\boldsymbol{B}_{\text {app }}\right|=20$, 40, 60, 80 and $100 \mathrm{mT}$ (middle); and $0.9 I_{\mathrm{c}}$ for $\left|\boldsymbol{B}_{\text {app }}\right|=20$, 40, 50 and $60 \mathrm{mT}$ (bottom). The data from such waveforms can then be transformed using (8)-(11) to calculate the dynamic resistance in $\left(\Omega \mathrm{m}^{-1} /\right.$ cycle $)$. Interestingly, figure 6 shows that dynamic resistance is incurred when $\mathrm{d}\left|\boldsymbol{B}_{a}(t)\right| / \mathrm{d} t$ is largest, i.e., in the region around $\boldsymbol{B}_{a}(t)=0$. In contrast, fluxflow resistance manifests itself as a spike in the voltage when $\mathrm{d}\left|\boldsymbol{B}_{a}(t)\right| / \mathrm{d} t$ is lowest, i.e., at the maxima and minima of the applied field, $\pm B_{\max }$. Additionally, there is some noticeable asymmetry of the voltage between positive and negative halfcycles of the applied field. This is related to the angular 
(a)
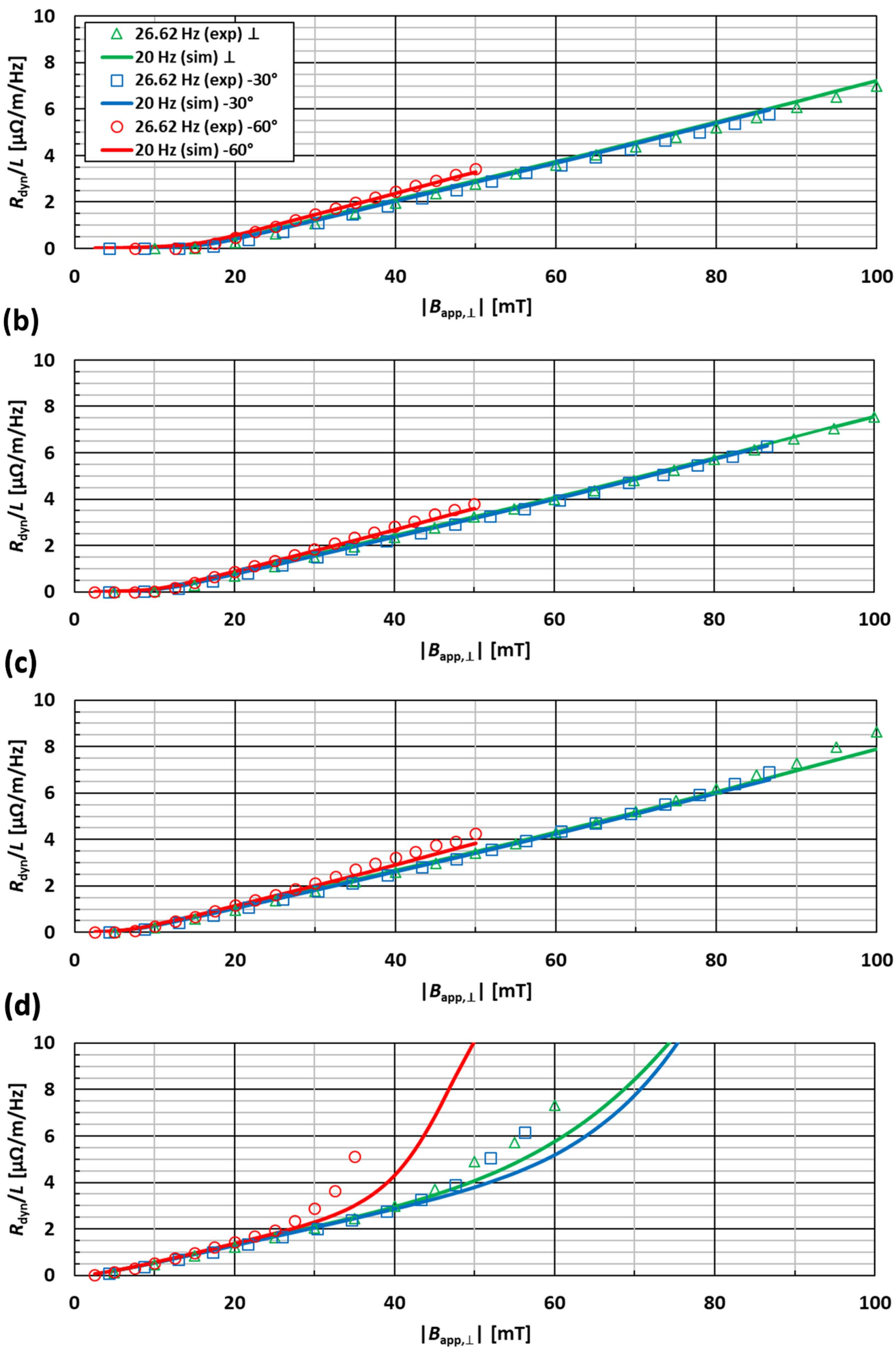

Figure 11. Comparison of the calculated dynamic resistance and experimental results for $I_{\mathrm{DC}} / I_{\mathrm{c}}=$ (a) 0.3 , (b) 0.5 , (c) 0.7 and (d) 0.9 for external AC magnetic field amplitudes up to $100 \mathrm{mT}$ (in $5 \mathrm{mT}$ increments and $f=20 \mathrm{~Hz}$ (simulation) and $26.62 \mathrm{~Hz}$ (experiment)) applied perpendicularly and at angles of $\theta=-30^{\circ}$ and $-60^{\circ}$. The results are plotted as a function of the amplitude of the perpendicular component of the applied magnetic field, $\boldsymbol{B}_{\text {app }, \perp}=\boldsymbol{B}_{\text {app }} \cdot \cos (\theta)$.

dependence of the superconducting properties of the wire (see figures 2 and 3), which differs slightly depending on whether the field is oriented towards the top surface $\left(\theta=0^{\circ}\right)$ or bottom surface (substrate side; $\theta=180^{\circ}$ ). For the wire studied here (for fields up to $100 \mathrm{mT}$ ), we see that $J_{\mathrm{c}}(B, \theta=$ $\left.180^{\circ}\right)$ tends to be slightly lower than $J_{\mathrm{c}}\left(B, \theta=0^{\circ}\right)$.

Figure 7 shows a comparison of the calculated dynamic resistance per cycle with the experimental results for 
(a)
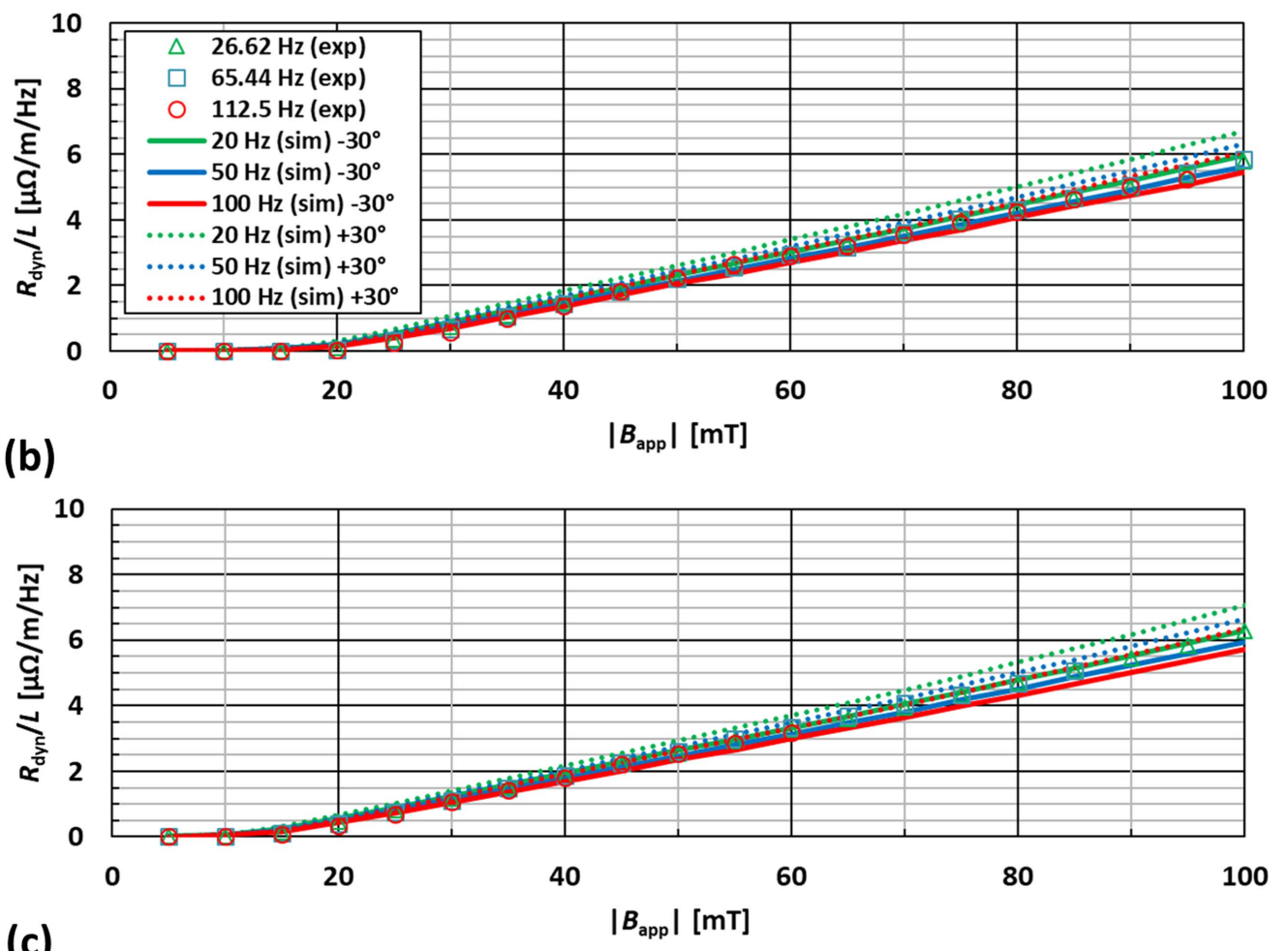

(c)

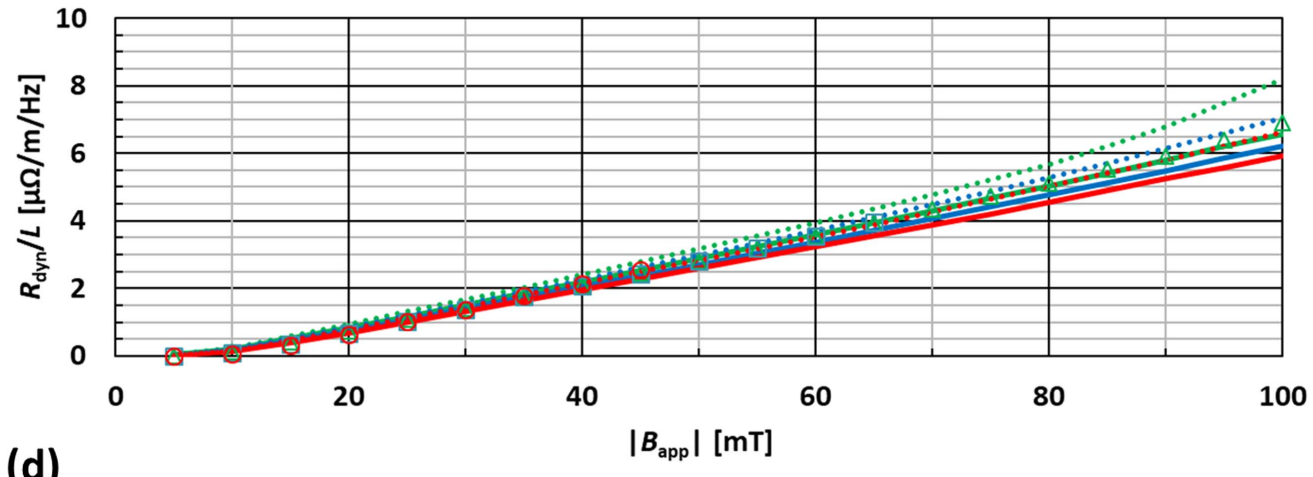

(d)

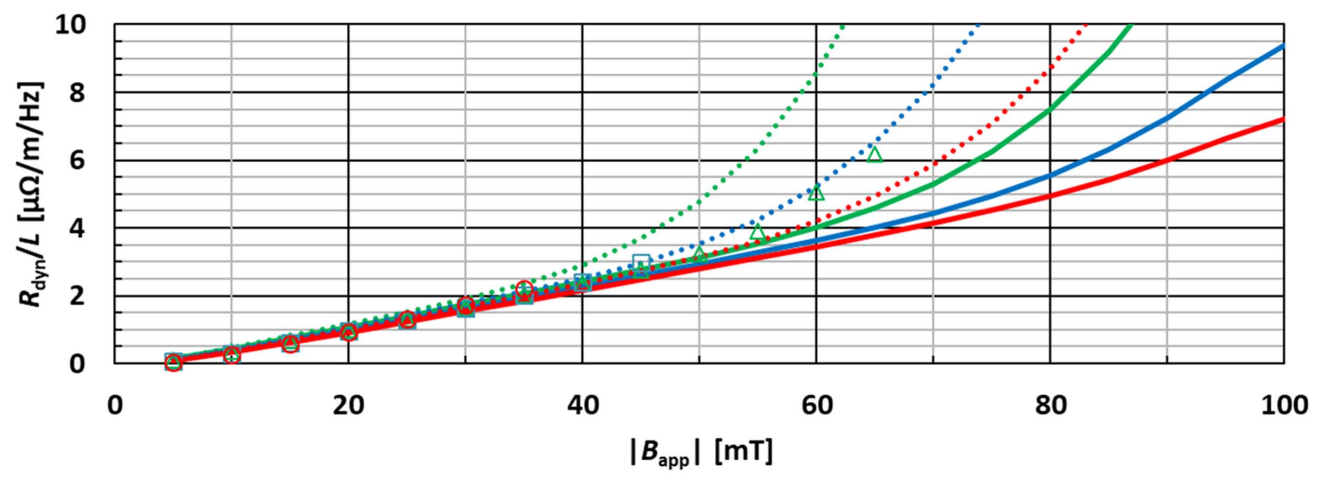

Figure 12. Comparison of the calculated dynamic resistance and experimental results for $I_{\mathrm{DC}} / I_{\mathrm{c}}=$ (a) 0.3 , (b) 0.5 , (c) 0.7 and (d) 0.9 for external AC magnetic field amplitudes up to $100 \mathrm{mT}$ (in $5 \mathrm{mT}$ increments) applied at an angle of $\theta= \pm 30^{\circ}$. The results are calculated for frequencies of 20,50 and $100 \mathrm{~Hz}$ and the experiments correspond to frequencies of 26.62, 65.44 and $112.5 \mathrm{~Hz}$.

$I_{\mathrm{DC}} / I_{\mathrm{c}}=$ (a) 0.3 , (b) 0.5 , (c) 0.7 and (d) 0.9 under applied perpendicular magnetic fields up to $100 \mathrm{mT}$ (in $5 \mathrm{mT}$ increments). The numerical results are calculated for frequencies of 20,50 and $100 \mathrm{~Hz}$ whilst the experimental results correspond to frequencies of 26.62, 65.44 and $112.5 \mathrm{~Hz}$. In figure $7(\mathrm{~d})$, the calculated results using the analytical equations presented in [30] are also included (closed symbols), which takes into account the flux-flow 
(a)
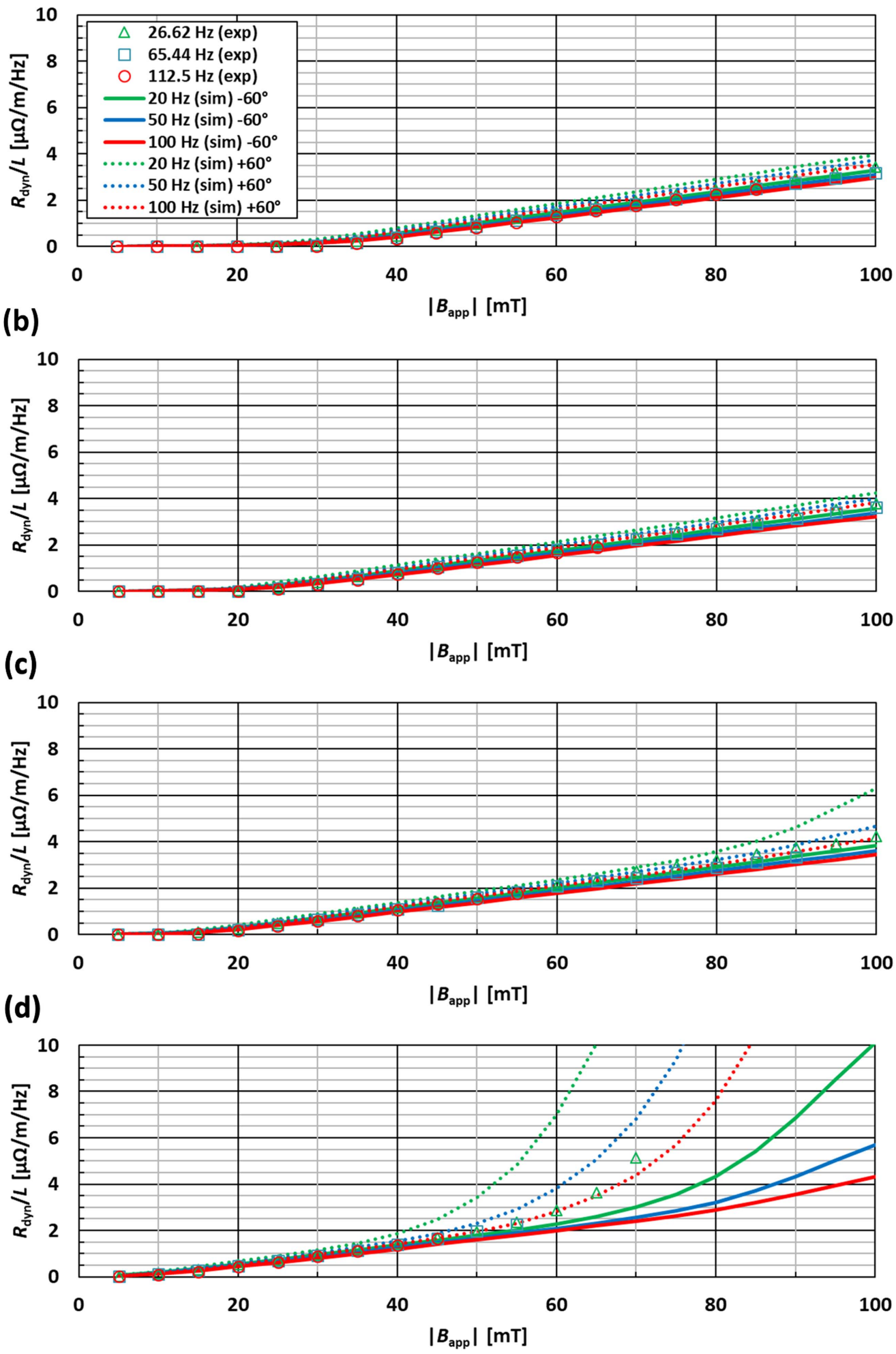

Figure 13. Comparison of the calculated dynamic resistance and experimental results for $I_{\mathrm{DC}} / I_{\mathrm{c}}=$ (a) 0.3 , (b) 0.5 , (c) 0.7 and (d) 0.9 for external AC magnetic field amplitudes up to $100 \mathrm{mT}$ (in $5 \mathrm{mT}$ increments) applied at an angle of $\theta= \pm 60^{\circ}$. The results are calculated for frequencies of 20,50 and $100 \mathrm{~Hz}$ and the experiments correspond to frequencies of 26.62, 65.44 and $112.5 \mathrm{~Hz}$.

resistance term that arises once the total driven transport current exceeds the field-dependent $I_{\mathrm{c}}$ of the wire. This effect gives rise to a nonlinear 'lift-off' from the constant value of $\mathrm{d} R_{\mathrm{dyn}} / \mathrm{d}\left|\boldsymbol{B}_{\text {app }}\right|$ observed at lower fields. The modelling results show excellent qualitative agreement with the experimental results and very good quantitative agreement, especially with respect to $B_{\text {th }}$ (the threshold field above which $R_{\text {dyn }}$ is generated) and the slope of $R_{\mathrm{dyn}}$. Three important points to 
(a)
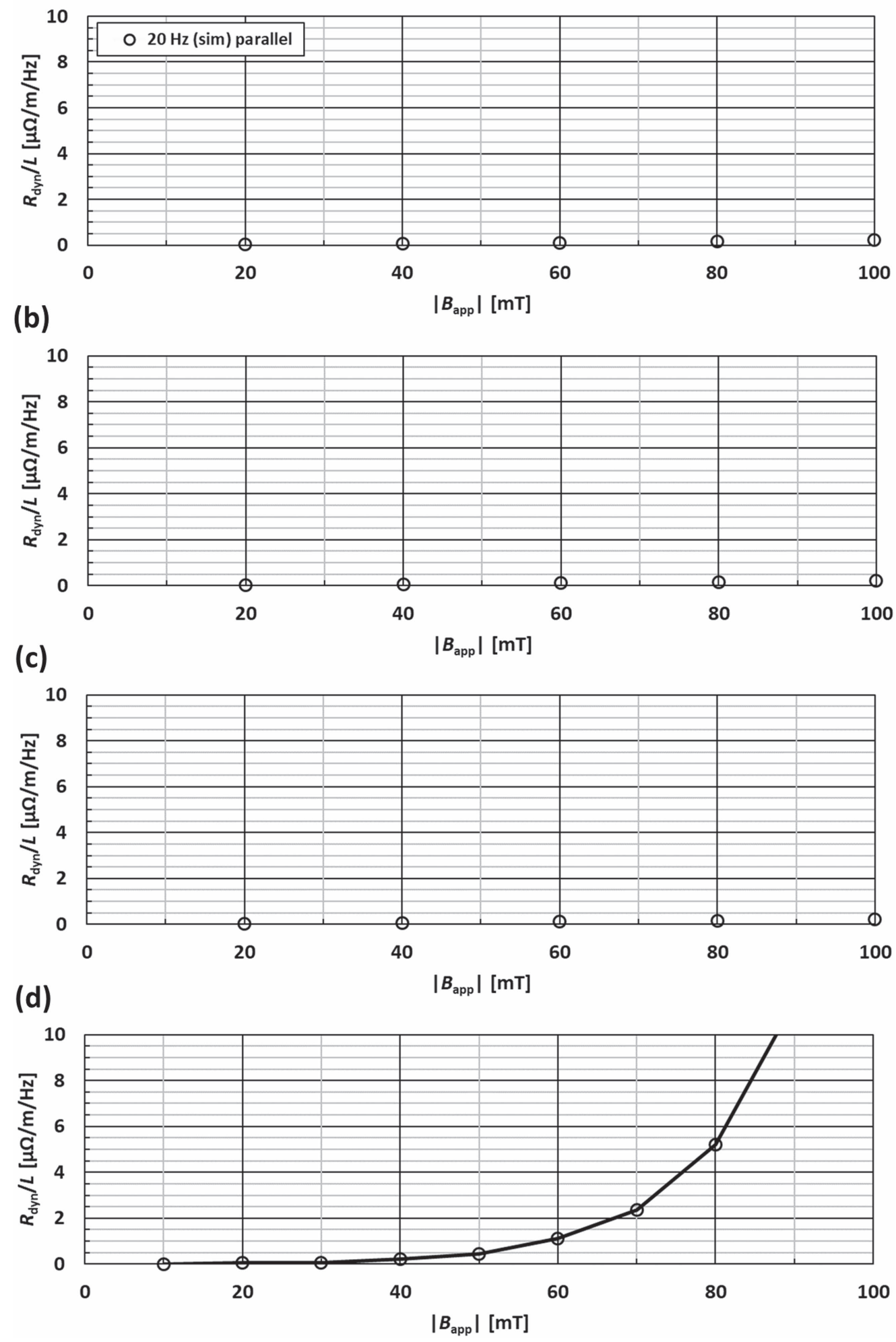

Figure 14. Calculated dynamic resistance for $I_{\mathrm{DC}} / I_{\mathrm{c}}=$ (a) 0.3 , (b) 0.5 , (c) 0.7 and (d) 0.9 for parallel magnetic fields (at an angle $\theta= \pm 90^{\circ}$ ) up to $100 \mathrm{mT}$ (in $20 \mathrm{mT}$ increments for (a)-(c) and $10 \mathrm{mT}$ increments for (d); $f=20 \mathrm{~Hz}$ ).

make are: (a) the calculated values exhibit a small frequency dependence of the slope, $\mathrm{d} R_{\mathrm{dyn}} / \mathrm{d}\left|\boldsymbol{B}_{\text {app }}\right|$, due to the use of the $E-J$ power law (as described earlier with reference to the AC loss results shown in figures 4 and 5); (b) isothermal conditions are assumed with a constant temperature,
$T=77 \mathrm{~K}$, so this ignores any temperature increase that might occur due to heat dissipation (in particular when the $J_{\mathrm{c}}$ of the wire is exceeded resulting in the 'lift-off' regime); and (c) the wire is assumed to have uniform properties across the entire cross-section (and along the length, which is a 
necessary condition for the 2D infinitely long approximation). Real wires do not entirely meet this criteria, and this can influence the DC and AC properties of an HTS coatedconductor wire [31, 41, 42]. In principle such information could also be implemented in the model to improve the modelling assumptions, as shown in [40]. Nevertheless, the numerical model developed here now provides a useful and flexible tool to predict the characteristics of dynamic resistance generated in any HTS coated-conductor wire for which the relevant geometric and superconducting properties are known, as well as more complicated arrangements of such wires, such as multiple-wire stacks [43], cables and so on.

In figure 5, the AC loss maxima are indicated for each DC transport current case. These correspond to the threshold field, $B_{\mathrm{th}}$, below which it is energetically favourable for the central region of the wire to be shielded from changes in the applied field [30]. When $B_{\mathrm{th}}$ is exceeded, dynamic resistance is incurred and increases linearly with $\left|\boldsymbol{B}_{\text {app }}\right|$. Using the numerical model, it is easy to visualise this effect, through mapping the current density distribution within the wire cross-section for different regimes under a perpendicular external AC magnetic field. Figure 8 shows the calculated current density distribution, normalised by $J_{\mathrm{c}}(\boldsymbol{B})$ (where $\boldsymbol{B}$ is the local magnetic flux density), for the simple DC transport current cases (in the absence of an applied AC field) at the time when the ramped current reaches its final value, $I_{\mathrm{DC}} / I_{\mathrm{c}}$. Figure 9 then shows the normalised, calculated current density distribution, which arises under a perpendicular external AC magnetic field when $I_{\mathrm{DC}}=0.3 I_{\mathrm{c}}$, for $\left|\boldsymbol{B}_{\text {app }}\right|=10 \mathrm{mT}$ (figure 9(a)) and $60 \mathrm{mT}$ (figure 9(b)). The distributions are shown at the positive and negative peaks of the applied field, as well as the zero-crossing ( $f=20 \mathrm{~Hz}$ ). Figure 9(a) corresponds to $R_{\text {dyn }}=0$ in figure 7(a), and shows the superposition of the transport current and magnetisation currents (with respect to figure 8). It clearly shows a shielded central region that does not experience a change in applied field, and for which $J=0$. In contrast, figure 9(b) shows the situation when the central region is no longer shielded throughout the entire cycle, such that $J \geqslant J_{\mathrm{c}}$ at all points within the wire, except for a very thin boundary region in which the current reverses direction. The result in this case is that a net flow of flux crosses the DC current-carrying region [4], leading to the development of $R_{\mathrm{dyn}}$.

In contrast, figure 10 shows the normalised current density distribution, $J / J_{\mathrm{c}}(\boldsymbol{B})$ when $I_{\mathrm{DC}}=0.9 I_{\mathrm{c}}$ for $\left|\boldsymbol{B}_{\text {app }}\right|=30 \mathrm{mT}$ (figure 10(a)) and $60 \mathrm{mT}$ (figure 10(b)). As before, the distributions are shown at the positive and negative peaks of the applied field, as well as the zerocrossing $(f=20 \mathrm{~Hz}$ ). The top figure corresponds to the linear regime (no shielded region and $\pm J_{\mathrm{c}}$ current-carrying regions). The bottom figure corresponds to the 'lift-off' regime (see figure $7(d)$ ), where there exists an additional flux-flow resistance term because the total driven transport current exceeds the field-dependent $I_{\mathrm{c}}$ of the wire. This 'lift-off' also results in the minima observed in the calculated total AC loss (see figure 5) and the development of a 'spike' in the calculated average electric field (see figure 6) around the peak of $\left|\boldsymbol{B}_{\text {app }}\right|$, which is in clear contrast to figure 6 (middle), where the average electric field is close to zero around the peak of $\left|\boldsymbol{B}_{\text {app }}\right|$.

\subsection{Angular dependence of dynamic resistance:}

$\theta= \pm 30^{\circ}, \pm 60^{\circ}$

The flexibility of the numerical model and the inclusion of the full angular dependence of the superconducting properties of the wire allows us to simulate the angular dependence of the dynamic resistance over the full range of applied field angles. Recent experimental measurements on a similar wire presented in [31] have shown that the dynamic resistance is dominated by the perpendicular magnetic field component, with negligible contribution from the parallel component. Figure 11 shows a comparison of the calculated dynamic resistance and experimental results for $I_{\mathrm{DC}} / I_{\mathrm{c}}=$ (a) 0.3 , (b) 0.5, (c) 0.7 and (d) 0.9 for magnetic fields up to $100 \mathrm{mT}$ (in $5 \mathrm{mT}$ increments and $f=20 \mathrm{~Hz}$ (simulation) and $26.62 \mathrm{~Hz}$ (experiment)) applied perpendicularly and at angles of $\theta=-30^{\circ}$ and $-60^{\circ}$. These results are plotted as a function of the amplitude of the perpendicular component of the applied magnetic field, $\boldsymbol{B}_{\text {app }, \perp}=\boldsymbol{B}_{\text {app }} \cdot \cos (\theta)$. This clearly shows that the dynamic resistance is dominated by the perpendicular component of the applied magnetic field, with some subtle differences: for $\theta=-60^{\circ}$, the slope $\mathrm{d} R_{\mathrm{dyn}} / \mathrm{d}\left|\boldsymbol{B}_{\mathrm{app}, \perp}\right|$ is slightly higher and 'lift-off' occurs at a lower value of $\left|\boldsymbol{B}_{\text {app }, \perp}\right|$.

To explain these subtle differences and to obtain a complete picture of the dynamic resistance of the wire, figure 12 shows a comparison of the angular dependence of the calculated dynamic resistance and experimental results for $I_{\mathrm{DC}} / I_{\mathrm{c}}=$ (a) 0.3 , (b) 0.5 , (c) 0.7 and (d) 0.9 for magnetic fields up to $100 \mathrm{mT}$ (in $5 \mathrm{mT}$ increments) applied at an angle of $\theta= \pm 30^{\circ}$, and figure 13 shows the same plots, but for an applied field angle of $\theta= \pm 60^{\circ}$. The numerical results are calculated for frequencies of 20,50 and $100 \mathrm{~Hz}$ and the experimental results correspond to frequencies of 26.62, 65.44 and $112.5 \mathrm{~Hz}$. The modelling results show excellent qualitative agreement with the experimental results and very good quantitative agreement, with the same comments to be made with respect to the observed quantitative discrepancies, i.e., a small frequency dependence due to the $E-J$ power law.

The results clearly show the influence of the asymmetric angular dependence of $J_{\mathrm{c}}$ and $n$ on the dynamic resistance. Figures 12 and 13 show that positive (dotted lines) and negative (solid lines) field angles result in different slopes of $R_{\text {dyn }}$ in the linear regime and a different onset of 'lift-off' behaviour (for $I=0.9 I_{\mathrm{c}}$ ). These asymmetric effects are caused by the different values of $J_{\mathrm{c}}$ and $n$ at $+|\theta|$ and $-|\theta|$ respectively. For example, and referring back to figure 2 , the $J_{\mathrm{c}}$ of the wire at $\theta=+30^{\circ}$ (and $\theta=+210^{\circ}$ for the negative half-cycle of the applied field) is comparatively lower than at $\theta=-30^{\circ}$ (i.e., at $\theta=+150^{\circ}$ and $+330^{\circ}$ in figure 2). Hence, the slope of $R_{\text {dyn }}$ is higher for $\theta=+30^{\circ}$, and the 'lift-off' criteria is satisfied at a lower value of $\left|\boldsymbol{B}_{\text {app }}\right|$. The same is true for the $\pm 60^{\circ}$ results shown in figure 12 .

In summary, the dynamic resistance in an HTS coatedconductor wire can be mostly determined by the perpendicular 
field component with subtle differences determined by the angular dependence of the superconducting properties of the wire, which can be included in detail within the numerical modelling framework.

\subsection{Dynamic resistance for parallel applied magnetic fields: $\theta= \pm 90^{\circ}$}

Finally, in this section, the dynamic resistance in parallel applied magnetic fields (at an angle $\theta= \pm 90^{\circ}$ ) is simulated using the same numerical framework. Figure 14 shows the calculated dynamic resistance for $I_{\mathrm{DC}} / I_{\mathrm{c}}=$ (a) 0.3 , (b) 0.5 , (c) 0.7 and (d) 0.9 for parallel magnetic fields up to $100 \mathrm{mT}$ (in $20 \mathrm{mT}$ increments for (a)-(c) and $10 \mathrm{mT}$ increments for (d), and $f=20 \mathrm{~Hz}$ ). There is negligible dynamic resistance generated until the total driven transport current exceeds $I_{\mathrm{c}}$ of the wire (in this case, when $I_{\mathrm{DC}} / I_{\mathrm{c}}=0.9$ and $\left|\boldsymbol{B}_{\text {app }}\right|$ is about $40 \mathrm{mT}$ or higher), resulting in the 'lift-off' regime explained earlier. This provides further evidence that the dynamic resistance in an HTS coated-conductor wire is determined mostly by the perpendicular component of the applied magnetic field and agrees with experimental measurements presented in [31]. This is in direct contrast with BSCCO wires $[4,44]$. From the viewpoint of practical applications, it is therefore the perpendicular component of any external, timevarying magnetic field that is of most interest, having a dominant impact on the generated dynamic resistance.

\section{Conclusion}

In this paper, a 2D numerical model based on the finiteelement method and implementing the $\boldsymbol{H}$-formulation is used to calculate the dynamic resistance and total AC loss in a coated-conductor HTS wire carrying an arbitrary transport current and exposed to background AC magnetic fields up to $100 \mathrm{mT}$. The measured angular dependence of the superconducting properties of wire are used as input data, and the model is validated using measured experimental data for magnetic fields perpendicular to the plane of the wire, as well as at angles of $\theta=30^{\circ}$ and $60^{\circ}$.

The model provides important insights into the origin and characteristic behaviour of dynamic resistance, including its relationship with the applied current and field, the wire's superconducting properties, the threshold field and the onset of nonlinear flux-flow resistance. The key conclusions of the paper can be summarised as follows.

(1) The normalised total AC loss of the superconducting wire (which includes contributions from both the magnetisation loss, $Q_{\mathrm{mag}}$, and the dynamic resistance, $\left.Q_{\text {dyn }}\right)$ exhibits particular maxima and minima turning points. The maxima correspond to the threshold field, $B_{\text {th }}$, above which there is no longer a shielded region inside the wire and dynamic resistance occurs, and these maxima shift to lower values of applied field with increasing $I_{\mathrm{DC}}$. The minima observed at higher transport currents relates to the emergence of an additional nonlinear flux-flow resistance term that arises once the total driven transport current exceeds the field-dependent $I_{\mathrm{c}}$ of the wire.

(2) The dynamic resistance is calculated from the average electric field across the entire conductor cross-section. The time-dependent waveform of this average electric field shows that dynamic resistance is incurred when $\mathrm{d}\left|\boldsymbol{B}_{a}(t)\right| / \mathrm{d} t$ is largest, i.e., in the region around $\boldsymbol{B}_{a}(t)=0$. In contrast, the generation of the flux-flow resistance manifests itself as a spike in the voltage when $\mathrm{d}\left|\boldsymbol{B}_{a}(t)\right| / \mathrm{d} t$ is lowest, i.e., at the maxima and minima of the applied field, $\pm B_{\max }$. Additionally, there is some noticeable asymmetry of the voltage between halfcycles related to the angular dependence of the superconducting properties of the wire.

(3) Calculated values of the dynamic resistance as a function of applied field angle show that the dynamic resistance can be mostly determined by the perpendicular field component. However, our calculations indicate subtle angular asymmetries that have not previously been experimentally reported. These are determined by the angular dependence of the superconducting properties of the wire. We also observe that the dynamic resistance under parallel applied fields is essentially negligible until $J_{\mathrm{c}}$ is exceeded and flux-flow resistance occurs.

This numerical modelling framework provides a useful and flexible tool to predict the characteristics of dynamic resistance generated in any HTS coated-conductor wire for which the relevant geometric and superconducting properties are known, as well as the possibility of assessing the emergence and impact of dynamic resistance in more complicated arrangements of conductors, such as multiple-wire stacks, cables and coils.

\section{Acknowledgments}

The authors kindly thank Dr Stuart Wimbush, Robinson Research Institute, Victoria University of Wellington, for the $I_{\mathrm{c}}(B, \theta)$ and $n(B, \theta)$ measurements of the wire. Mark Ainslie would like to acknowledge financial support from an Engineering and Physical Sciences Research Council (EPSRC) Early Career Fellowship EP/P020313/1. Additional data related to this publication are available at the University of Cambridge data repository (https://doi.org/10.17863/CAM. 20647). This work was also supported in part by the Japan Society for the Promotion of Science (JSPS).

\section{ORCID iDs}

Mark D Ainslie (i) https://orcid.org/0000-0003-0466-3680

Chris W Bumby (iD https://orcid.org/0000-0001-8555-2469

Zhenan Jiang (10) https://orcid.org/0000-0002-3482-3510

Naoyuki Amemiya (iD https://orcid.org/0000-0002-

3000-864X 


\section{References}

[1] Andrianov V V, Zenkevitch V B, Kurguzov V V, Sytchev V V and Ternovskii F F 1970 Sov. Phys._JETP 31815

[2] Ogasawara K, Yasukochi K, Nose S and Sekizawa H 1976 Cryogenics 1733

[3] Ogasawara K, Takahashi Y, Kanbara K, Kubota Y, Yosohama K and Yasukochi K 1979 Cryogenics 19736

[4] Oomen M P, Rieger J, Leghissa M, ten Haken B and ten Kate H H J 1999 Supercond. Sci. Technol. 12382

[5] Geng J and Coombs T A 2015 Appl. Phys. Lett. 107142601

[6] Geng J, Matsuda K, Fu L, Fagnard J-F, Zhang H, Zhang X, Shen B, Dong Q, Baghdadi M and Coombs T A 2016 J. Phys. D: Appl. Phys. 49 11LT01

[7] Jiang Z, Hamilton K, Amemiya N, Badcock R A and Bumby C W 2014 Appl. Phys. Lett. 105112601

[8] Jiang Z, Bumby C W, Badcock R A, Sung H J, Long N J and Amemiya N 2015 Supercond. Sci. Technol. 28115008

[9] Bumby C W, Jiang Z, Storey J G, Pantoja A E and Badcock R A 2016 Appl. Phys. Lett. 108122601

[10] Kajikawa K, Hayashi T, Yoshida R, Iwakuma M and Funaki K 2003 IEEE Trans. Appl. Supercond. 133630

[11] Pecher R, McCulloch M D, Chapman S J, Prigozhin L and Elliott C M 2003 Proc. 6th EUCAS pp 1-11

[12] Hong Z, Campbell A M and Coombs T A 2006 Supercond. Sci. Technol. 191246

[13] Brambilla R, Grilli F and Martini L 2007 Supercond. Sci. Technol. 2016

[14] Ainslie M D, Flack T J, Hong Z and Coombs T A 2011 Int. J. Comput. Math. Electr. Electron. Eng. 30762

[15] Ainslie M D, Yuan W, Hong Z, Pei R, Flack T J and Coombs T A 2011 IEEE Trans. Appl. Supercond. 213265

[16] Ainslie M D, Flack T J and Campbell A M 2012 Physica C 47250

[17] Liu G, Zhang G, Yu H, Jing L, Ai L and Liu Q 2017 J. Appl. Phys. 121243902

[18] Liu G, Zhang G, Jing L, Yu H, Ai L, Yuan W and Li W 2017 IEEE Trans. Appl. Supercond. 276603807

[19] Plummer C J G and Evetts J E 1987 IEEE Trans. Magn. MAG-23 1179

[20] Rhyner J 1993 Physica C 212292

[21] Zermeno V M R, Habelok K, Stepien M and Grill F 2017 Supercond. Sci. Technol. 30034001

[22] HTS Modeling Workgroup 2013 Shared models (http:// htsmodelling.com/?page_id=748)

[23] Zermeno V M R, Abrahamsen A B, Mijatovic N, Jensen B B and Sorensen M P 2013 J. Appl. Phys. 114173901
[24] Zermeno V M R and Grill F 2014 Supercond. Sci. Technol. 27 044025

[25] Hu D, Ainslie M D, Rush J P, Durrell J H, Zou J, Raine M J and Hampshire D P 2015 Supercond. Sci. Technol. 2865001

[26] Hu D, Ainslie M D, Raine M J, Hampshire D P and Zou J 2016 IEEE Trans. Appl. Supercond. 266600906

[27] Ainslie M D, Rodriguez-Zermeno V M, Hong Z, Yuan W, Flack T J and Coombs T A 2011 Supercond. Sci. Technol. 24045005

[28] Ainslie M D, Hu D, Zou J and Cardwell D A 2015 IEEE Trans. Appl. Supercond. 254602305

[29] Grilli F, Zermeno V M R, Pardo E, Vojenciak M, Brand J, Kario A and Goldacker W 2014 IEEE Trans. Appl. Supercond. 244801005

[30] Jiang Z, Toyomoto R, Amemiya N, Zhang X and Bumby C W 2017 Supercond. Sci. Technol. 30 03LT01

[31] Jiang Z, Zhou W, Li Q, Yao M, Fang J, Amemiya N and Bumby C W 2018 Supercond. Sci. Technol 31035002

[32] Li Q, Yao M, Jiang Z, Bumby C W and Amemiya N 2018 IEEE Trans. Appl. Supercond. 286600106

[33] Jiang Z, Toyomoto R, Amemiya N, Bumby C W, Badcock R A and Long N J 2017 IEEE Trans. Appl. Supercond. 275900205

[34] Jiang Z and Amemiya N 2004 Supercond. Sci. Technol. 17 371-9

[35] Fukui S, Kitoh Y, Numata T, Tsukamoto O, Fujikami J and Hayashi K 1998 Adv. Cryogenic Eng. 44723

[36] Brandt E H and Indenbom M 1993 Phys. Rev. B 4312893

[37] Sander M and Grilli F 2010 J. Phys.: Conf. Ser. 234022030

[38] Thakur K P, Raj A, Brandt E H and Pamidi S V 2011 Supercond. Sci. Technol. 24045006

[39] Thakur K P, Raj A, Brandt E H, Kvitkovic J and Pamidi S V 2011 Supercond. Sci. Technol. 24065024

[40] Grilli F, Pardo E, Stenvall A, Nguyen D N, Yuan W and Gomory F 2014 IEEE Trans. Appl. Supercond. 24 8200433

[41] Gomory F, Souc J, Pardo E, Seiler E, Soloviov M, Frolek L, Skarba M, Konopka P, Pekarcikova M and Janovec J 2013 IEEE Trans. Appl. Supercond. 235900406

[42] Hu D, Ainslie M D, Zou J and Cardwell D A 2015 IEEE Trans. Appl. Supercond. 254900605

[43] Jiang Z, Zhou W, Bumby C W, Staines M, Li Q, Badcock R A, Long N J and Fang J 2018 IEEE Trans. Appl. Supercond. 28 8200305

[44] Ciszek M, Knoopers H G, Rabbers J J, ten Haken B and ten Kate H H J 2002 Supercond. Sci. Technol. 151275 